\title{
WestVirginiaUniversity
}

THE RESEARCH REPOSITORY @ WVU

Graduate Theses, Dissertations, and Problem Reports

2012

\section{Sol-Gel Derived Wear-Resistant, Hydrophobic, Particle-Reinforced Silica Coatings}

Derrick A. Banerjee

West Virginia University

Follow this and additional works at: https://researchrepository.wvu.edu/etd

\section{Recommended Citation}

Banerjee, Derrick A., "Sol-Gel Derived Wear-Resistant, Hydrophobic, Particle-Reinforced Silica Coatings" (2012). Graduate Theses, Dissertations, and Problem Reports. 4829.

https://researchrepository.wvu.edu/etd/4829

This Thesis is protected by copyright and/or related rights. It has been brought to you by the The Research Repository @ WVU with permission from the rights-holder(s). You are free to use this Thesis in any way that is permitted by the copyright and related rights legislation that applies to your use. For other uses you must obtain permission from the rights-holder(s) directly, unless additional rights are indicated by a Creative Commons license in the record and/ or on the work itself. This Thesis has been accepted for inclusion in WVU Graduate Theses, Dissertations, and Problem Reports collection by an authorized administrator of The Research Repository @ WVU. For more information, please contact researchrepository@mail.wvu.edu. 
Sol-Gel Derived Wear-Resistant, Hydrophobic, Particle-Reinforced Silica Coatings

Derrick A. Banerjee

Thesis submitted to the

Benjamin M. Statler College of Engineering and Mineral Resources at West Virginia University in partial fulfillment of the requirements

for the degree of

\author{
Master of Science \\ in \\ Mechanical Engineering \\ Darran R. Cairns, Ph.D., Chair \\ Konstantinos A. Sierros, Ph.D. \\ Stephen N. Kukureka, Ph.D.
}

Department of Mechanical and Aerospace Engineering

\author{
Morgantown, West Virginia
}

2012

Keywords: sol-gel, silica, tribology, wear, hydrophobic, coatings

Copyright 2012 Derrick A. Banerjee 


\section{ABSTRACT \\ Sol-Gel Derived Wear-Resistant, Hydrophobic, Particle-Reinforced Silica Coatings \\ Derrick A. Banerjee}

Hydrophobic coatings are useful for a wide range of applications including anti-fouling, anti-corrosive, and anti-icing. There are also a number of emerging applications in which transparency is critical such as optoelectronics, touchscreens, and smart windows. For many of these applications, resistance to abrasive wear is also important. Typical low-surface energy coatings such as polytetrafluoroethylene (PTFE) have poor mechanical strength which leaves them susceptible to abrasive wear. To combat this problem, functional moieties can be encapsulated using a sol-gel method to provide improved substrate adhesion and hardness while maintaining transparency and functionality. While this provides an improvement for many applications, the inherent porosity from this approach has a detrimental effect on the overall performance of the coating. A solution to this is to provide a nanoparticle-reinforced sol-gel matrix to improve the hardness and abrasive wear resistance. These coatings can improve the lifetime and performance of the aforementioned applications by protecting them from the harsh environments typically encountered.

Silica nanoparticle-reinforced fluorinated silica coatings were deposited by sol-gel synthesis on glass substrates via dip coating. Varying amounts of colloidal silica nanoparticles from 0.5 to 10 weight percent of precursor were added. Structural, mechanical, surface, functional, and tribological properties were examined to elucidate the effects of the nanoparticles on the silica matrix. A lab-built reciprocating abrasive wear apparatus, a reciprocating tribometer, contact angle goniometry, nanoindentation, nano-scratch, atomic force microscopy, and stylus profilometry were used to investigate these properties. Experimental results show that the particle-reinforced coatings provide increased overall indentation hardness as well as a decreased wear rate while maintaining comparable functional sustainability to coatings without the added silica particles. 


\section{Acknowledgements}

I would like to express my deepest gratitude to all of these people who have in some way contributed to the publication of this Master's thesis:

My research advisor and committee chair, Dr. Darran Cairns, for his continuous support, enthusiasm, and knowledge. Without the opportunities he has provided, I would not be where I am today.

Committee member, Dr. Konstantinos Sierros for his dedication, encouragement, help, and motivation over the years. Dr. Sierros' support for my research and career has been immeasurable.

Committee member, Dr. Stephen Kukureka, for being an excellent academic grandfather and having mentored Dr. Cairns and Dr. Sierros to be great researchers and advisors in their own rights.

Dr. Aaron Kessman, for his invaluable guidance every step along the way. His research at WVU set the foundation for everything I've done.

My incredible labmates (and friends): Ed Chambers, Sean Cronin, Nick Morris, Emmie DeFusco, Teddy Bejitual, and Andrew Hoover; for their contributions, support, and camaraderie. I have been extremely lucky to be able to work with and look forward to continuing working with all of you.

Everyone at CSM Instruments: Dr. Nicholas Randall, Rahul Nair, Dr. Bo Zhou, and Drew Griffin; for their assistance and expertise with their equipment and for the invaluable experience I had in Boston with them.

Last but not least I want to thank all of my other friends and family, especially my fiancé Devin and my parents Rob and Kathy for their unwavering support and love. 


\section{Table of Contents}

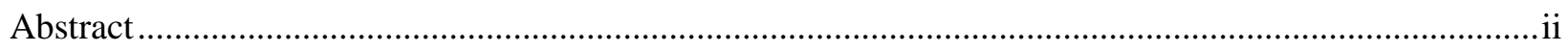

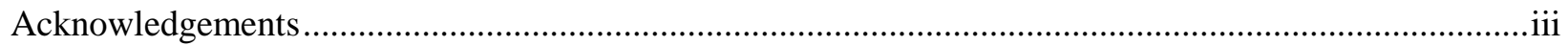

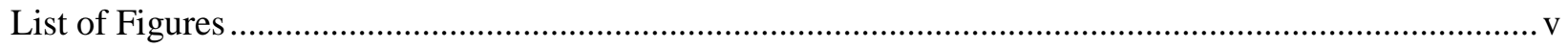

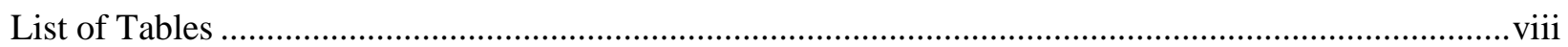

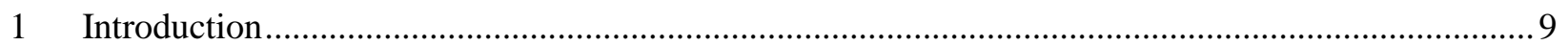

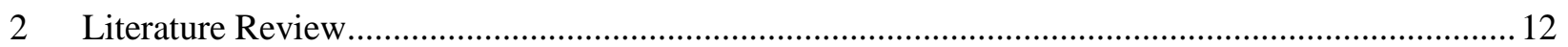

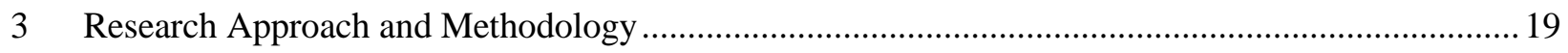

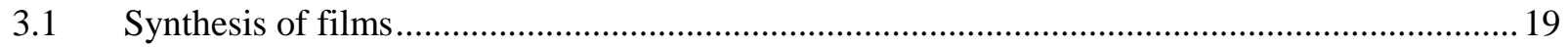

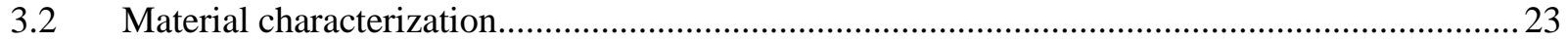

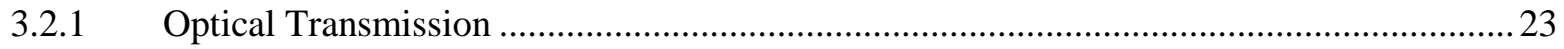

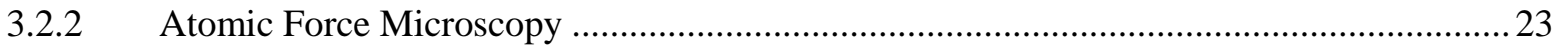

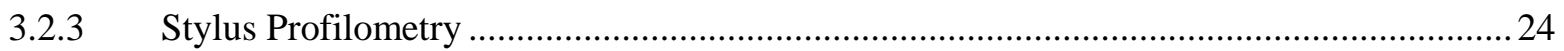

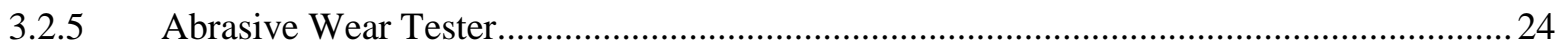

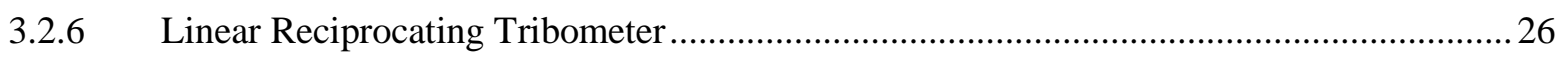

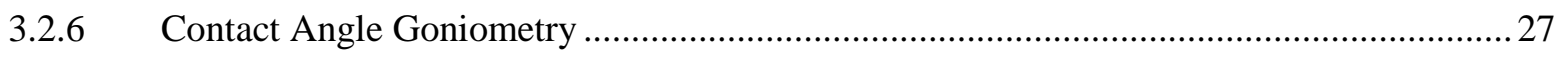

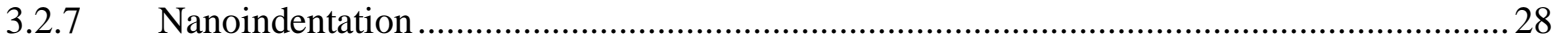

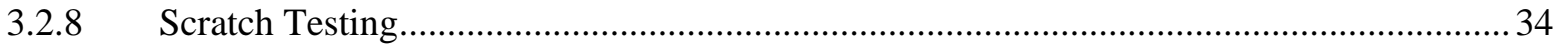

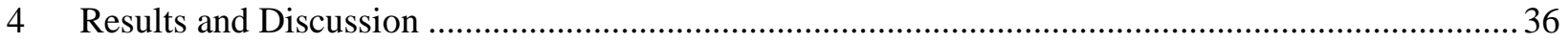

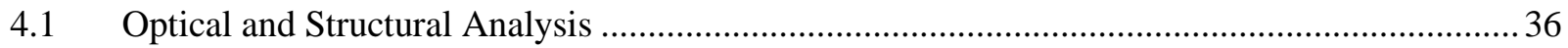

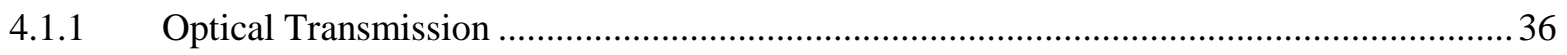

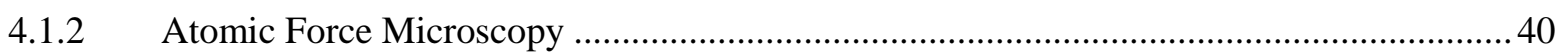

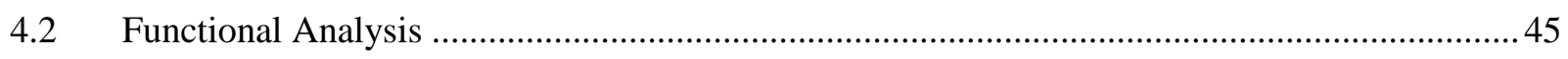

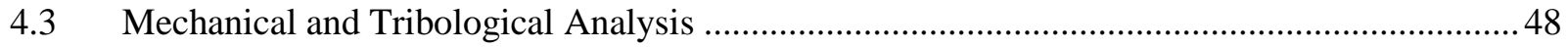

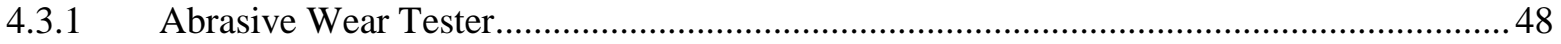

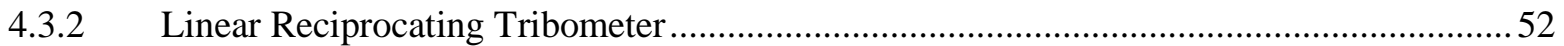

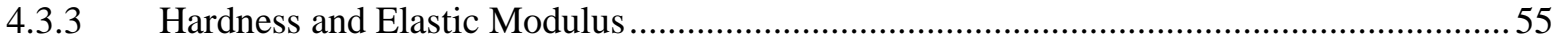

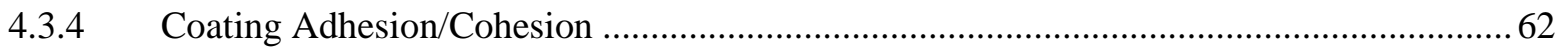

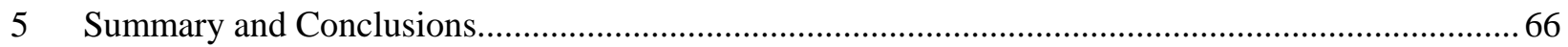

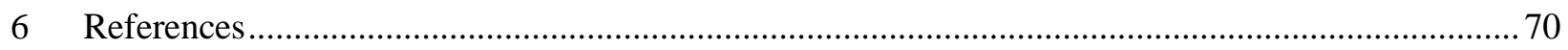




\section{List of Figures}

Figure 1: Schematic of nanoparticle-reinforced functional sol-gel coating.

Figure 2: Water contact angle of films with $0 \%$ and $8 \%$ surfactant concentration vs. number of wear cycles [11] 15

Figure 3: Wear rate at varying concentrations of fluorosilanes and surfactant [7] 16

Figure 4: Nanoindendation hardness of films synthesized with different fluorosilane concentrations and with and without surfactant [7] 16

Figure 5: Chemical structure of PFPE Fluorolink S10 [2] 19

Figure 6: Flow chart of sol-gel synthesis of hydrophobic, particle-reinforced silica coatings ..... 20

Figure 7: Reciprocating polishing wear apparatus schematic [32]..... 25

Figure 8: Linear-reciprocating Micro-Tribometer (CSM Instruments) ……………………....... 27

Figure 9: Schematic of a cross-section through an indentation [36] ......................................... 29

Figure 10: Schematic of load vs. displacement curve for an indent [36] ................................... 30

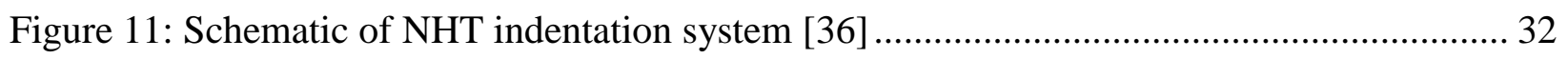

Figure 12: Sample setup under UNHT Ultra Nanoindentation Tester ........................................ 33

Figure 13: Sample setup under NHT Nanoindentation Tester..................................................... 34

Figure 14: Sample setup under Nano-scratch Tester ................................................................ 35

Figure 15: Optical transmission spectrum for non-templated coating with no added silica.......... 37

Figure 16: Optical transmission spectrum for non-templated coating with 1\% added silica ........ 37

Figure 17: Optical transmission spectrum for non-templated coating with $2 \%$ added silica ........ 38

Figure 18: Optical transmission spectrum for non-templated coating with 5\% added silica ........ 38

Figure 19: Optical micrographs of non-templated coatings with (a) $0 \%$, (b) $1 \%$, (c) $2 \%$, and (d) $5 \%$ added colloidal silica 39 
Figure 20: AFM tapping mode micrograph of a non-templated coating with $2 \%$ added colloidal silica 40

Figure 21: Topography cross section using AFM through a non-templated coating with 2\% added colloidal silica corresponding to the green line from Figure 20. 41

Figure 22: AFM tapping mode micrograph of a non-templated coating with $3 \%$ added colloidal silica. 42

Figure 23: Topography cross section using AFM through a non-templated coating with 3\% added colloidal silica corresponding to the green line from Figure 22. 42

Figure 24: AFM contact mode micrographs of a templated coating with $2 \%$ added colloidal silica. (a) Topographical mapping and (b) friction force mapping. 43

Figure 25: Topography cross section using AFM through a templated coating with $2 \%$ added colloidal silica corresponding to the green line from Figure 24.

Figure 26: Examples of water droplet contact angles on (a) a pristine surface, (b) a partially worn surface, and (c) bare glass. 45

Figure 27: Contact angle of non-templated coatings vs. the penetration depth into the coating as a percent of the initial film thickness. 47

Figure 28: Wear rate calculated from mass loss of non-templated coatings with added colloidal silica 49

Figure 29: Wear rate of templated coatings with added colloidal silica using the Archard equation...... 51

Figure 30: Coefficient of friction output vs. wear cycles of non-templated coating with $2 \%$ added colloidal silica using CSM Instruments Micro-Tribometer 53 
Figure 31: Optical micrographs of wear tracks of non-templated coatings with (a) $0 \%$ and (b) $2 \%$ added colloidal silica. 54

Figure 32: Representative normal load-penetration depth curve of a standard indentation protocol performed on particle-reinforced coatings with UNHT 55

Figure 33: Indentation hardness and elastic moduli of non-templated coatings....................... 57

Figure 34: Indentation hardness and wear rates of non-templated coatings ............................ 58

Figure 35: Indentation hardness and wear rates of templated coatings .................................. 59

Figure 36: Indentation curve for a continuous multicycle indentation protocol on a non-templated coating with $2 \%$ added colloidal silica 60

Figure 37: Hardness of a non-templated $2 \%$ added colloidal silica coating as a function of penetration depth into the coating. 61 Figure 38: Panoramic optical micrographs of nanoscratch test on (a) $0 \%$, (b) $1 \%$, (c) $2 \%$ added colloidal silica. Arrows signify critical failure loads. 63 Figure 39: Optical micrograph of (a) $\mathrm{L}_{\mathrm{C} 1}$ and (b) $\mathrm{L}_{\mathrm{C} 2}$ of a non-templated coating without added silica. 63

Figure 40: Optical micrograph of (a) $\mathrm{L}_{\mathrm{C} 1}$ and (b) $\mathrm{L}_{\mathrm{C} 2}$ of a non-templated coating with $1 \%$ added colloidal silica. 64

Figure 41: Optical micrograph of $\mathrm{L}_{\mathrm{C} 1}$ of a non-templated coating with $2 \%$ colloidal silica. 65 


\section{List of Tables}

Table 1: Coating formulations for non-templated and templated coatings............................. 21

Table 2: Added DP5820 colloidal silica mass for different concentrations ............................. 22

Table 3: Specifications of the CSM Instruments' NHT and UNHT ....................................... 31 


\section{Introduction}

The objective of this research was to develop wear-resistant, hydrophobically functional coatings using a sol-gel processing method and to investigate their mechanical, functional, and tribological properties. Other current and potential applications for protective durable functional coatings include anti-fouling, anti-microbial [1,2], anti-icing [3], and anti-corrosive coatings [4]. The ultimate goal for designing mechanically robust functional coatings for the widest range of applications is to have high hardness and wear resistance. However, there tends to be a compromise between mechanical properties and other requirements such as substrate independence, thermal stability, optical transparency, and coating thickness. For instance, typical high hardness coatings are not transparent and require expensive and undesirable deposition techniques. Thus, the realistic goal is to optimize the coatings such that they have the highest hardness and best wear resistance possible without compromising other essential characteristics.

Typical hydrophobically functional coatings are based on organic molecules that suffer from inherent mechanical weaknesses which results in loss of functionality in practical abrasive applications [5]. Soft polymeric functional materials such as polytetrafluoroethylene (PTFE) also suffer from low adhesive strength to substrates [6]. A common approach taken to offset these weaknesses is through a sol-gel process of co-condensation of silicon alkoxides and fluorosilanes to provide a low-surface energy interface. This process provides a strong $\mathrm{Si}-\mathrm{O}$ film-substrate bond due to the sol-gel grafting [7]. However, the setback for this approach is that these fluorinated moieties are thermodynamically driven to the solid-vapor interface during deposition [8,9], leading to surface stratification and poor functionalization of the bulk coating. The top 
surface provides excellent functionality, but this molecularly thin monolayer also inherently has low abrasive resistance.

Kessman et al. $[5,7,10,11]$ have reported previously the synthesis of coatings which counteract surface stratification by encapsulating the functional fluorinated silanes within the bulk of the film via evaporation-induced assembly with a surfactant template. While this method produces an increased hydrophobicity within the bulk of the coating compared to non-templated coatings, there is an increase in film porosity. This increased porosity was shown to lower the hardness of the sol-gel films. It was shown that the wear rate was double that of any nontemplated coating of similar functional concentration due to this increase in porosity [7].

A solution to this problem is to incorporate a nanoparticle-reinforced sol-gel matrix to improve the hardness and abrasive wear resistance of the bulk coating, thus counteracting the detrimental effects of the increased porosity. A model of the structure of this coating is shown in Figure 1.

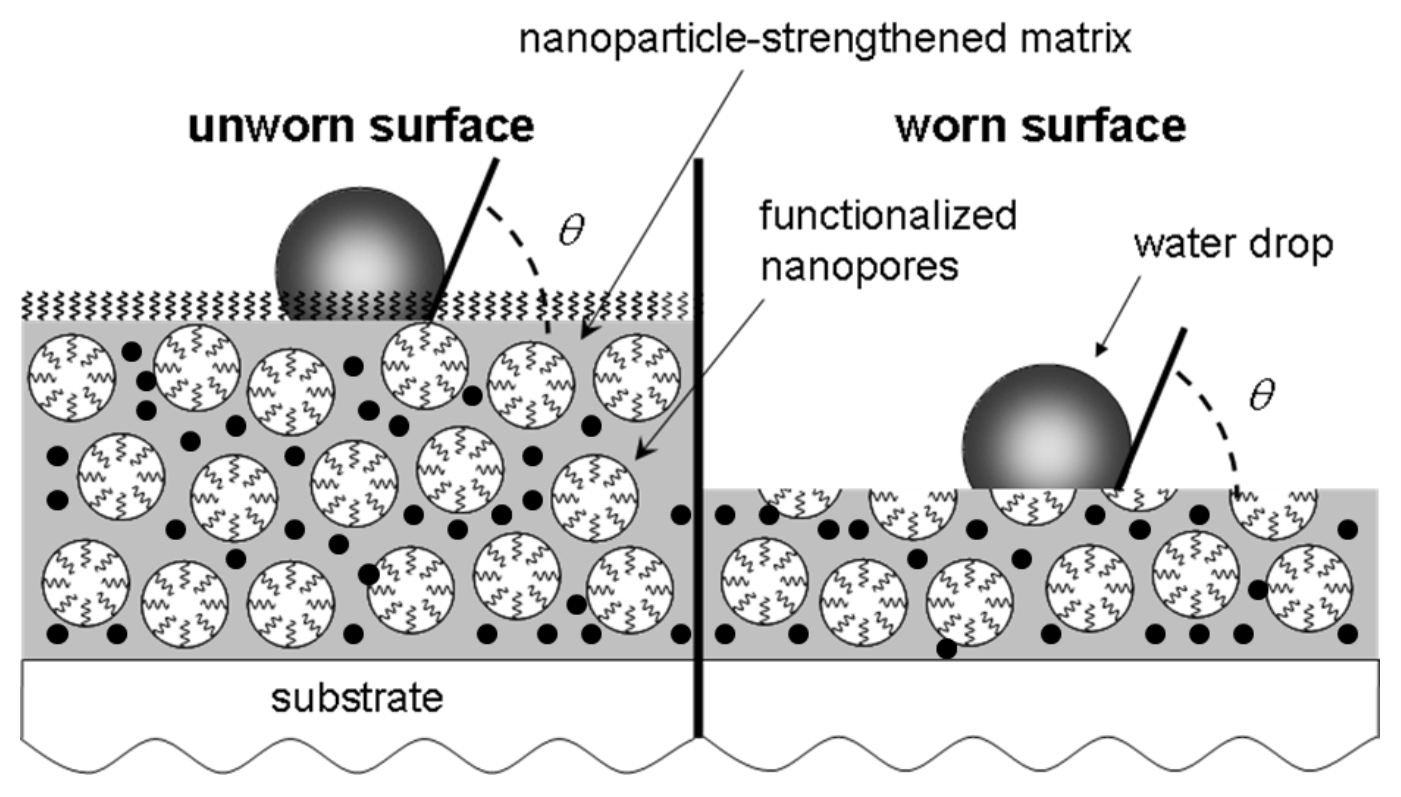

Figure 1: Schematic of nanoparticle-reinforced functional sol-gel coating 
It has been shown that ceramic colloidal additives can be used in self-assembly processes to improve overall film durability [12]. The addition of colloidal nanoparticles could also have the benefit of imparting a textural advantage to enhance the surface functional properties through increased roughness [13]. Though the optimum balance between the bulk hydrophobicity and wear resistance can be tailored through surfactant templating, the overall hardness of the matrix itself can be increased by inclusion of colloidal silica nanoparticles. The interplay between these parameters is central to this study.

For this project, hydrophobic functionality was used as a probe to investigate functional sustainability since other functionalities would require more complex analytical techniques. Additionally, the requirements for overcoming the surface-segregation of the fluorinated silanes will be more of a challenge than other organic functionalities [5]. Hydrophobic/non-wetting coatings are widely applicable as thin films on bulk substrates. Some of these applications include optoelectronics such as solar panels [15,16], information displays [17,18], optical filters [17], touchscreens, ophthalmic lenses [19], optical storage disks [20], painting glazing [21], industrial, automotive, textile, household applications [22], and architectural structures/urban infrastructure [23]. Incorporation of wear-resistant hydrophobic layers can reduce waste and cleaning needs and extend the lifetime of these applications. 


\section{Literature Review}

This section reviews relevant prior work and methods applied and adapted for the research investigation of wear-resistant functional coatings for a wide range of applications.

Current methods for imparting surface functionality generally fall under three main categories: (1) soft organic materials used as thick films, (2) biomimetic textured surfaces with high aspect ratio features, and (3) self-assembled surface active monolayers. In addition to these methods, some such as Kessman et al. have explored the use of a templated mesoporous structure that can encapsulate functionality. Each of these methods has inherent drawbacks when it comes to wear-resistant functionality, and all will be discussed briefly except the mesoporous method which will be more in depth.

In typical environments encountered, devices such as touchscreens and solar panels are susceptible to degradation through abrasive wear. Any coating used to protect these devices must be mechanically robust to withstand these tribological forces. Soft organic materials such as PTFE used as thin films will have inherently low abrasive wear resistance and substrate adhesion [6]. In an attempt to overcome this, they are often applied as thick films and sacrifice their optical properties. This is not desirable for many applications. To compound this problem, they are also relatively difficult to deposit on varieties of substrates.

Textured surfaces with high aspect ratio features are another way of imparting surface functionality. Experimentation by Hong et al. [24] involved a silica underlayer with a fluorocarbon layer and controlled silica sol aging to produce a high surface roughness. This surface roughness was supposed to be used as an "enhancement factor for chemical and mechanical durability" [24]. It was claimed that with average roughnesses from between 8 and 
$80 \mathrm{~nm}$, the abrasive wear rate of the coating could be decreased. The authors' did claim to sustain functionality after abrasive wear, but only to a surface roughness of about $10 \mathrm{~nm}$. Thus, the induced roughness as a way to provide larger surface area contact for fluorocarbon bonding was counterproductive under abrasive wear testing. Cui et al. [25] used various etching methods to induce surface roughness similar to the well-known Lotus effect. These methods do not produce functionally durable coatings. Rather they are simply surface pretreatments and do not depend on the coating composition or microstructure. The desire is to produce a substrateindependent coating that does not involve any chemical or physical roughening of the surface prior to deposition to develop a coating formulation that would work independently of the substrate condition since it is not always possible or desired to texture the substrate surface.

Yoneda and Morimoto [26] examined the use of a water-repellent fluorinated organosilane monolayer deposited on similarly treated surfaces to those of Hong and Cui. Through a controlled rubbing wear tester, this was found to be a sufficiently durable method for their purposes and it was concluded that the films had strong bonding and high adhesion density due to their roughed substrates. Giessler et al. [27] synthesized $10 \mathrm{~nm}$ thick layers on glass substrates. While there was some durability in the wear rates calculated for these coatings, the functionality suffered after initial abrasive wear. While these coatings can be strong adhesively, owing to the Si-O film-substrate bond [7] via sol-gel grafting, the fluorinated silanes are driven to the surface (the solid-vapor interface) thermodynamically. Thus the functionality is not sufficient within the bulk of the coating. This surface segregation is compounded by the weakness of the functional molecules at the surface.

The innovative route taken by Kessman et al. involved the use of a template-assisted encapsulation of fluorinated silanes within the sol-gel matrix $[5,7,10,11]$. This technique 
provided sustained hydrophobic and oleophobic (oil-repelling) functionality even after more abrasive wear. Unlike Hong et al. and Cui et al., the coatings synthesized were not substratedependent and did not require pre-roughening. This technique was reviewed previously by Raman, Anderson, and Brinker [28]. The overall structure of the coatings made by Kessman et al. were of the disordered structure as prescribed by Raman et al., but this was found to be somewhat beneficial through abrasive wear analysis.

Kessman et al. investigated the effect on the functional, mechanical, tribological, and structural properties of the coating by the addition of a block copolymer surfactant template $[5,7,10,11]$. Since sustained functionality was the overall goal, contact angle goniometry was used to determine the water and oil contact angles of the bulk of the coating and the surface energies associated with each. Additionally, XPS depth profiling was utilized to determine the chemical structure of the bulk to determine the extent of the fluorosilane encapsulation in the mesopores. Nitrogen adsorption porosimetry was used to quantify the effects of porosity induced by varying concentrations of surfactant. Likewise, varying levels of fluorosilane concentrations were used to elucidate their effects on the structure and functionality of the coatings. A mild abrasive wear tester was used to compare wear rates among the varying formulations of coatings. To compare with the wear testing, nanoindentation and nano-scratch testing were performed to further characterize properties and wear mechanisms.

Overall, Kessman et al. found that bulk functionality could be improved through this templated encapsulation of fluorinated silanes within the bulk of the coating, as shown in Figure 2. 


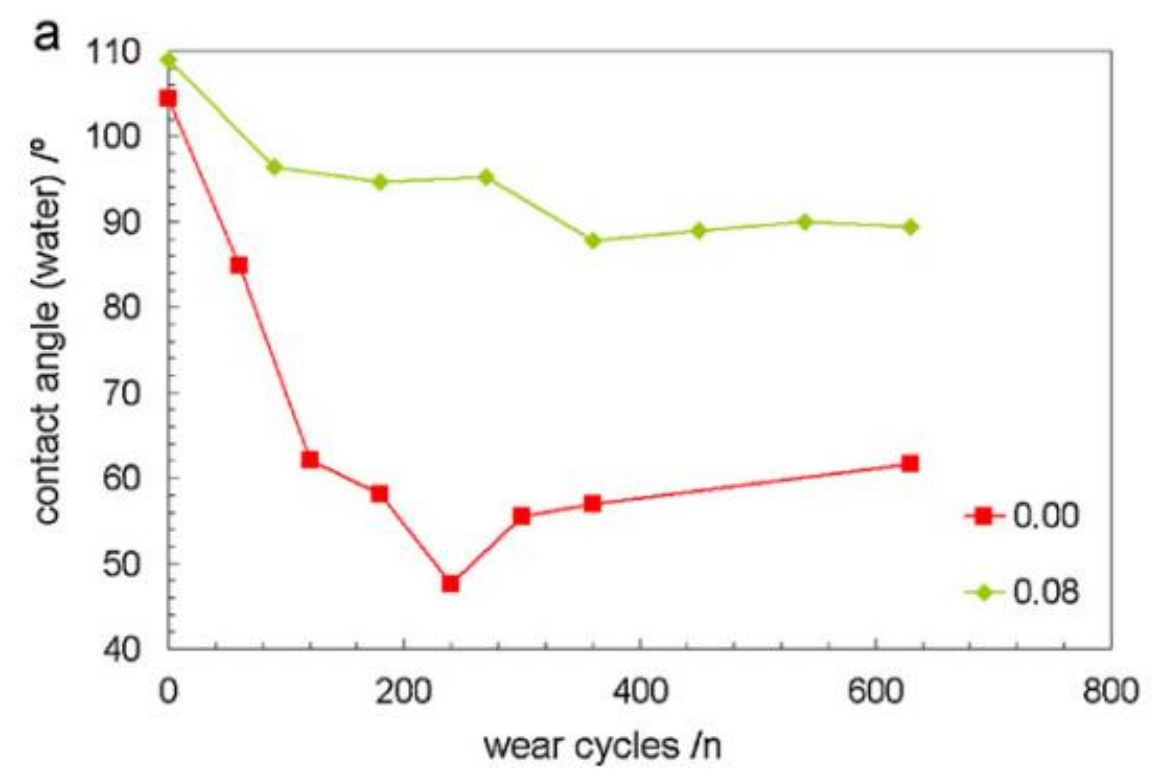

Figure 2: Water contact angle of films with $0 \%$ and $8 \%$ surfactant concentration vs. number of wear cycles [11]

However, it was found that this induced porosity would have a detrimental effect on the mechanical and tribological properties of the coatings. The wear rate increased, as shown in Figure 3, and the hardness decreased, as shown in Figure 4, with the addition of surfactant to the sol. Similarly, substrate adhesion decreased with the addition of surfactant. 


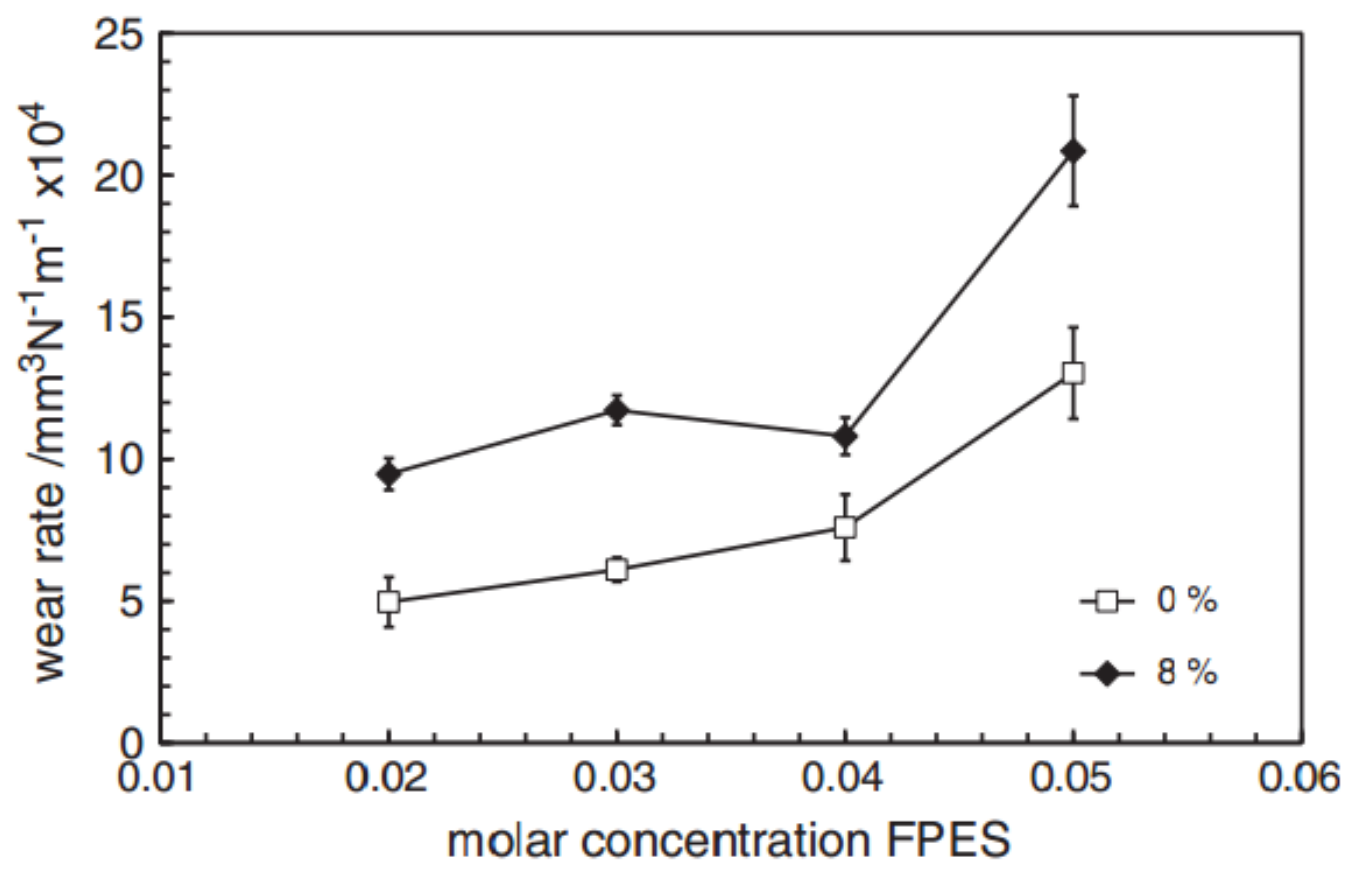

Figure 3: Wear rate at varying concentrations of fluorosilanes $(0.02-0.05)$ and surfactant $(0$ and $8 \%$ ) [7]

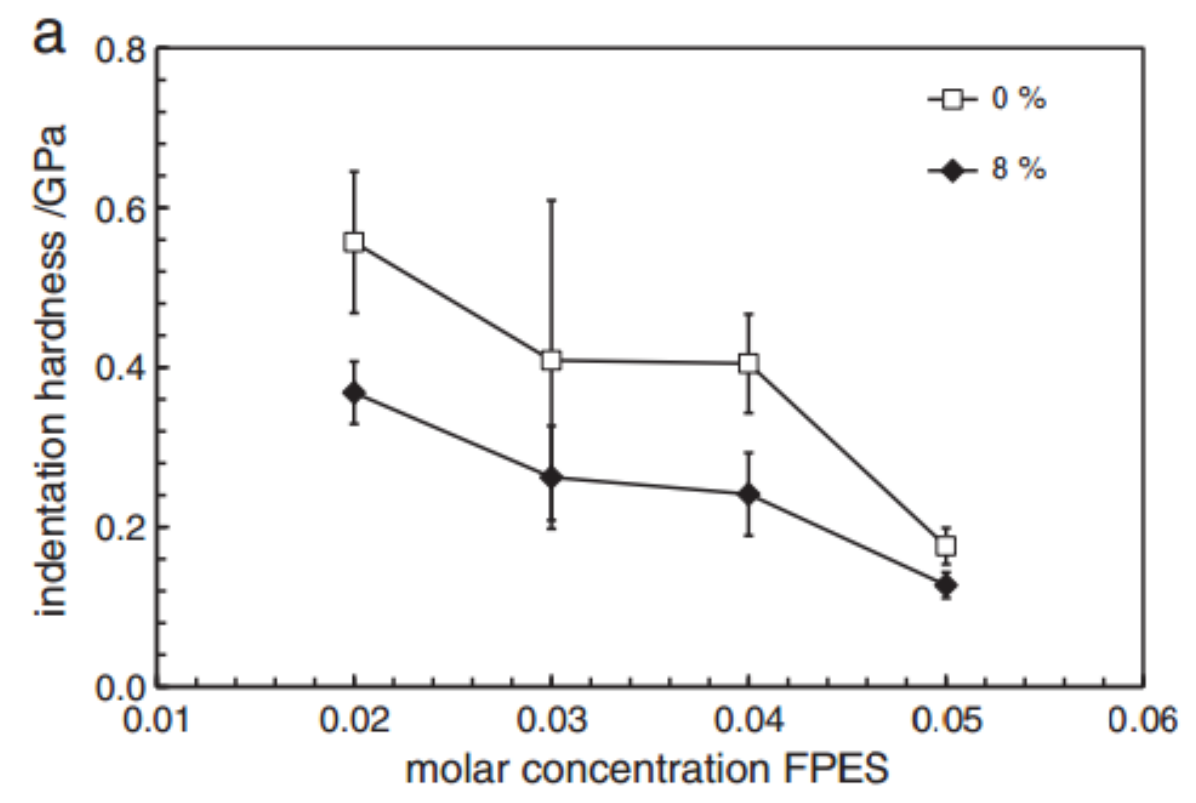

Figure 4: Nanoindendation hardness of films synthesized with different fluorosilane concentrations $(0.02-0.05)$ and with and without surfactant [7] 
The coatings synthesized by Kessman et al. maintained functionality but they were mechanically weakened after a point that this improvement could not be justified. However, an advantage of sol-gel processing is the ability to tailor the structure and composition of the films through chemistry. The optimum concentrations and molar ratios of fluorosilanes, surfactant, tetraethoxysilane precursor, and acid catalyst were determined through these mechanical and tribological characterizations.

One possible solution for enhancing the mechanical and tribological properties of these coatings is through reinforcement of the sol-gel matrix. López et al. [29] employed a 0.1 wt\% carbon nanotube reinforcement method. The coatings were tested using a dry sliding wear test and compared it to a typical coating without reinforcement. It was found that there was a significant decrease in specific wear rate with the addition of these carbon nanotubes into the solgel matrix by mechanical mixing.

Another possible route is through nanoparticle reinforcement. Suegama et al. [12] investigated the tribological behavior of organosilane films with added silica nanoparticles with an average particle size of $10 \mathrm{~nm}$. Differing concentrations of silica particles were used to determine their effect on the properties of the coating. They found an increased overall hardness using nanoindentation methods and quantified the surface agglomeration of the silica particles. They found an optimum silica concentration of $300 \mathrm{ppm}$ of the precursors.

Hwang et al. incorporated silica nanoparticles in a matrix of trimethoxysilane and tetramethyl orthosilicate [30]. These coatings consisted of a rough surface of a homogeneous monolayer with thickness of about $40 \mathrm{~nm}$. While this surface was similar to that of the Lotus leaf biomimicry as before, the approach taken by Hwang et al. was to characterize the abrasive wear 
resistance as a function of surface morphology. They found that the roughest surfaces had the highest abrasive wear rates.

Lakshmi et al. [31] used a hybrid sol of methyltriethoxysilane with colloidal silica with particle size between $25-30 \mathrm{~nm}$. These coatings were spray-coated and exhibited an extremely high water contact angle due to the roughness of the surface. The hardness was characterized using a pencil test and it was determined to exhibit a hardness of $5 \mathrm{H}$ or $6 \mathrm{H}$ depending on concentrations of silica. However, the coatings were not resistant to abrasive wear resistance. This study was more focused on the effects of hydrophobicity rather than the overall mechanical and tribological strength of the coating. 


\section{$3 \quad$ Research Approach and Methodology}

\subsection{Synthesis of films}

Synthesis of the sol-gel derived silica nanoparticle-reinforced fluorinated coatings follows the process outlined by Kessman et al. [5,7,10,11] with a few exceptions and alterations. The sol-gel process used involves the co-condensation of fluorinated silane with an alkoxide silica precursor. The alkoxide precursor used was tetraethoxysilane (TEOS, tetraethyl orthosilicate 98\%, Acros Organics), and the functional fluorinated silane used was a perfluoropolyether silane (PFPE, Fluorolink S10, Solvay Solexis, molecular weight $=1850$ $\mathrm{g} / \mathrm{mol}$ ). The chemical structure of this fluorosilane is shown in Figure 5.

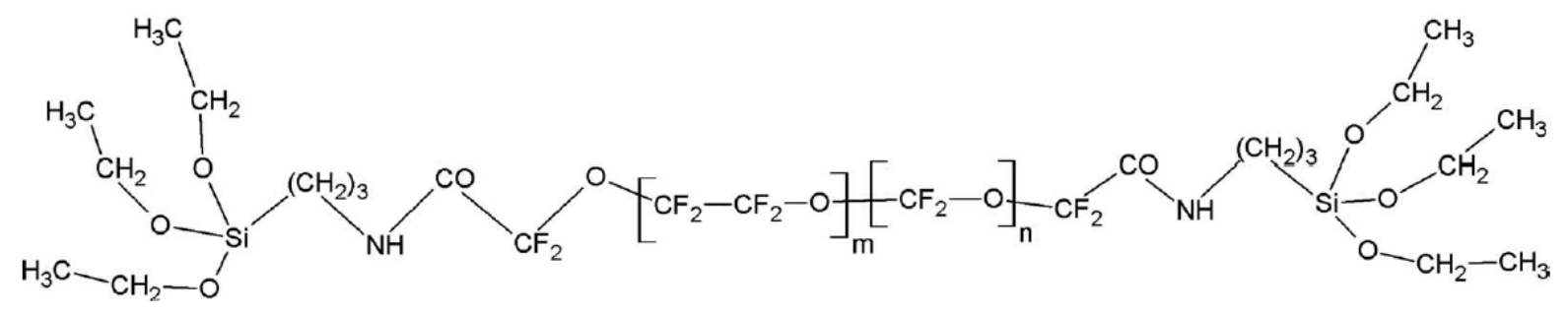

Figure 5: Chemical structure of PFPE Fluorolink S10. $\mathrm{m} / \mathrm{n}=1.5-2.5,2<\mathrm{n}<5,5<\mathrm{m}<9$ [2]

Some of the coatings were also templated with a surfactant after the initial sol synthesis. This surfactant, when used, was the block copolymer Pluronic F127 $\left(\mathrm{PEO}_{106} \mathrm{PPO}_{70} \mathrm{PEO}_{106}, \mathrm{BASF}\right.$, molecular weight $=12,600 \mathrm{~g} / \mathrm{mol}$ ). The added colloidal silica nanoparticles used were $20 \mathrm{~nm}$ in diameter dispersed in ethylene glycol at a concentration of 30\% (DP5820, Nyacol Nano Technologies). This particular particle size was chosen based on methods and results from literature [29-31]. 
The sol-gel solutions were synthesized in approximately 30-35 g batches in the following order and as shown in the flow chart in Figure 6.

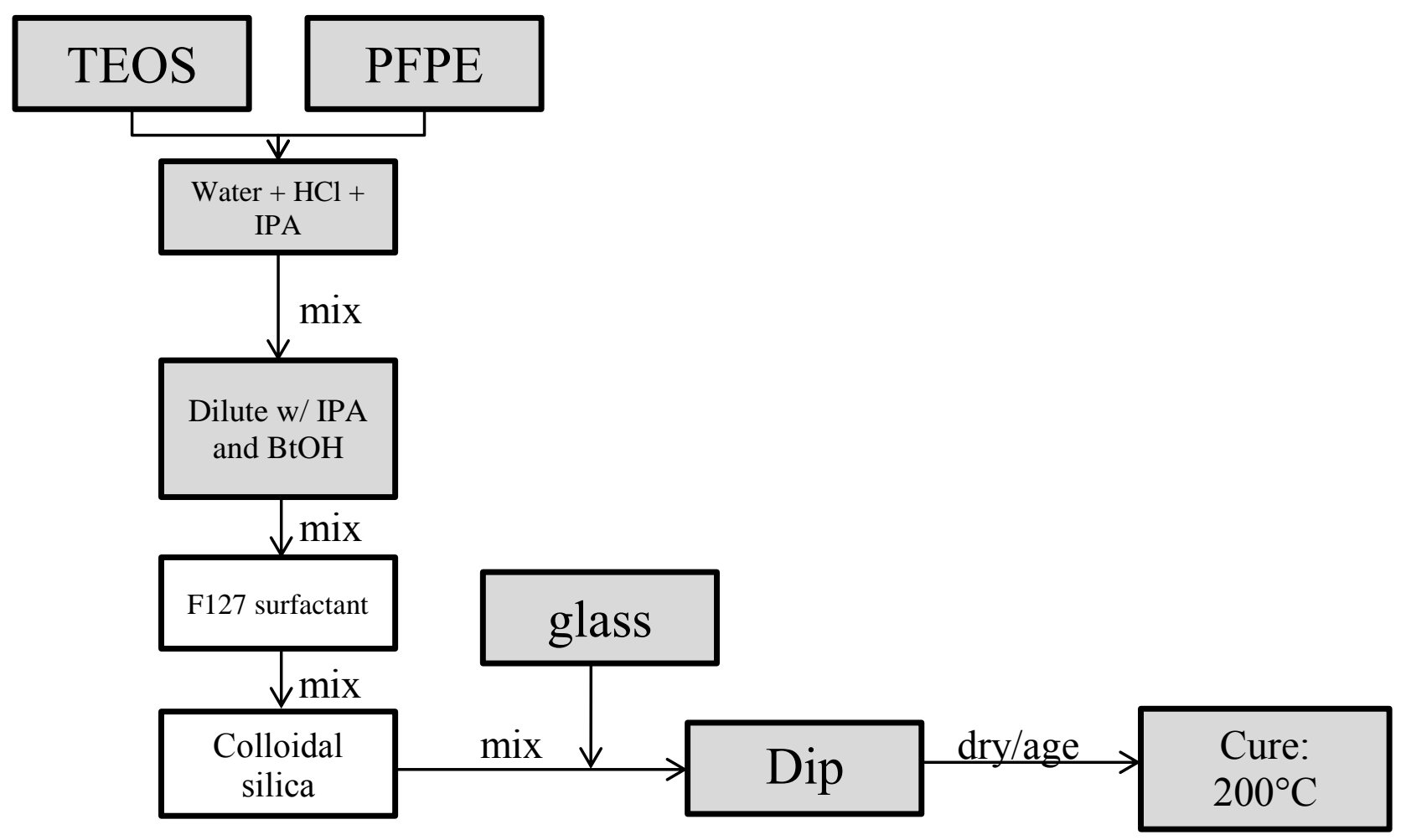

Figure 6: Flow chart of sol-gel synthesis of hydrophobic, particle-reinforced silica coatings TEOS and PFPE were hydrolyzed with water, isopropyl alcohol (IPA), and a small amount of hydrochloric acid $(\mathrm{HCl})$ in the molar ratio 0.98 TEOS : 0.02 PFPE $: 4 \mathrm{H}_{2} \mathrm{O}: 1 \mathrm{IPA}: 0.01 \mathrm{HCl}$ and stirred for 1 hour using a magnetic stirrer (VWR Professional Stirrer \#97042-706, VWR International, Radnor, PA). The sol was then diluted with IPA and butanol (BtOH) for a final molar ratio of 0.98 TEOS : 0.02 PFPE : $4 \mathrm{H}_{2} \mathrm{O}: 10$ IPA : $0.01 \mathrm{HCl}: 2 \mathrm{BtOH}$. For the surfactant templated coatings, the Pluronic F127 was added at a concentration calculated as 5 mass percent as established by Kessman et al. [5,7,10,11]. This concentration was calculated as follows:

$$
100 \% \times \frac{\mathrm{F} 127}{\mathrm{TEOS}+\mathrm{PFPE}+\mathrm{F} 127}
$$


These templated solutions were mixed for an additional hour. The next step was to add the colloidal silica at varying concentrations. These concentrations were calculated as a percent of the mass of the TEOS and PFPE used and varied from 0 to $10 \%$. After mixing for another 10 minutes, the sols were coated on soda-lime glass microscope slides by dip coating using a KSV Instruments dip coater with a withdrawal speed of $200 \mathrm{~mm} / \mathrm{min}$. All coatings were synthesized and deposited in controlled ambient conditions of $23 \pm 1^{\circ} \mathrm{C}$ and $40 \pm 10 \%$ relative humidity. After deposition, the films were dried for 24 hours at ambient conditions. Finally, the coatings were cured in a box furnace at $200^{\circ} \mathrm{C}$ for 2 hours with a ramp rate of $10^{\circ} \mathrm{C} / \mathrm{min}$ from ambient conditions. Coating formulations used are listed in TABLES 1 and 2

Table 1: Coating formulations for non-templated and templated coatings

\begin{tabular}{|c|c|c|c|}
\cline { 3 - 4 } \multicolumn{1}{c|}{} & moles & $\begin{array}{c}\text { Non- } \\
\text { Templated }\end{array}$ & Templated \\
\cline { 2 - 4 } \multicolumn{1}{c|}{} & 0.98 & mass $(\mathrm{g})$ & mass $(\mathrm{g})$ \\
\hline PFOS & 0.02 & 4.40 & 4.4 \\
\hline IPA & 10 & 0.797 & 0.797 \\
\hline Water & 4 & 12.952 & 12.952 \\
\hline HCl (3.7\% w/w & 0.01 & 0.2124 & 0.2124 \\
\hline in water) & 2 & 5.086 & 5.086 \\
\hline F1OH & $5 \mathrm{wt} \%$ & 0 & 0.274 \\
\hline
\end{tabular}


Table 2: Added DP5820 colloidal silica mass for different concentrations

\begin{tabular}{|c|c|}
\hline Concentration (wt\%) & added DP5820 (g) \\
\hline 0.5 & 0.0865 \\
\hline 1 & 0.173 \\
\hline 2 & 0.346 \\
\hline 3 & 0.519 \\
\hline 5 & 0.865 \\
\hline 10 & 1.73 \\
\hline
\end{tabular}




\subsection{Material characterization}

\subsubsection{Optical Transmission}

Optical transmission of the added colloidal silica sol-gel coatings was measured. Transmission was measured for the visible light spectrum between $380-750 \mathrm{~nm}$ and calculated as a percentage of the ambient transmission. Optical transmission was measured using a UV-vis spectrometer (Jaz Spectrometer Module, Ocean Optics, Inc., Dunedin, FL) with the deuteriumtungsten halogen light source module.

\subsubsection{Atomic Force Microscopy}

Atomic force microscopy (AFM) provided high resolution surface mapping for these coatings. AFM was performed using a Molecular Imaging PicoScan 3000 system in both tapping and contact modes. The contact mode tip (SC37B, MikroMasch) used was a silicon tip on a cantilever with force constant $\mathrm{k}=0.3 \mathrm{~N} / \mathrm{m}$ and radius of curvature less than $10 \mathrm{~nm}$. The tapping mode tip (ACT-W, Applied NanoStructures) used was also a silicon tip on a cantilever with

nominal $\mathrm{k}=50 \mathrm{~N} / \mathrm{m}$. and radius of curvature less than $10 \mathrm{~nm}$. Simultaneous topography and friction mapping were conducted in contact mode while tapping mode only provided topographical images. All measurements were conducted in controlled ambient conditions of $23 \pm 1^{\circ} \mathrm{C}$ and $40 \pm 10 \%$ relative humidity. 


\subsubsection{Stylus Profilometry}

Each coating thickness was measured on a Veeco Dektak 150 stylus profilometer (Bruker AXS, Tucson, AZ) by measuring the depth of a manual scratch through the center of the coating. The scratch was made using a razor blade such that the glass substrate was not affected. The Dektak 150 stylus used had a $12.5 \mu$ m radius tip with a nominal applied normal force of $0.1 \mathrm{mN}$. The highest resolution depth range was used for each scan at $6.5 \mu \mathrm{m}$. Average roughness values were also measured on the Veeco Dektak 150. The scan lengths used for roughness measurements were between 500 and $1000 \mu \mathrm{m}$.

\subsubsection{Abrasive Wear Tester}

In order to determine the coatings' resistance to abrasive wear, films were abraded using a lab-built reciprocating polishing wear device as shown in Figure 7. This instrument has been used previously for wear studies on sol-gel coatings [5,7,10,11,32]. The device provides a conformal surface that uniformly and gradually wears through coatings with the thicknesses on the order of several hundred nanometers in a controlled manner. Samples were periodically removed for analysis, before which they were cleaned with water and alcohol to remove residue from abrasion.

The instrument consists of a vertically-loaded reciprocating polishing contact controlled by a stepper motor (NM34A200, Zaber Technologies, Vancouver, BC). The coating abrasion occurs from the trapped $0.3 \mu \mathrm{m}$ alumina particles (product \#90-187120, Allied High Tech Products, Inc., Rancho Dominguez, CA) in a cloth pad (product \#90-150285, Allied). The alumina particles are suspended in an aqueous slurry that is regularly replenished, and the cloth 
pad is rotated after wear intervals to give a pristine surface for abrasion. The normal load was kept constant at $4.70 \mathrm{~N}$. The polishing contact was determined to be over a nominal area of 175 $\mathrm{mm}^{2}$ for an approximate Hertzian contact pressure of $25 \mathrm{kPa}$. The stroke length of the reciprocating contact was $35 \mathrm{~mm}$ at $0.5 \mathrm{~Hz}$. These parameters were chosen to model contact wear on a touchscreen or cleaning of solar panels.

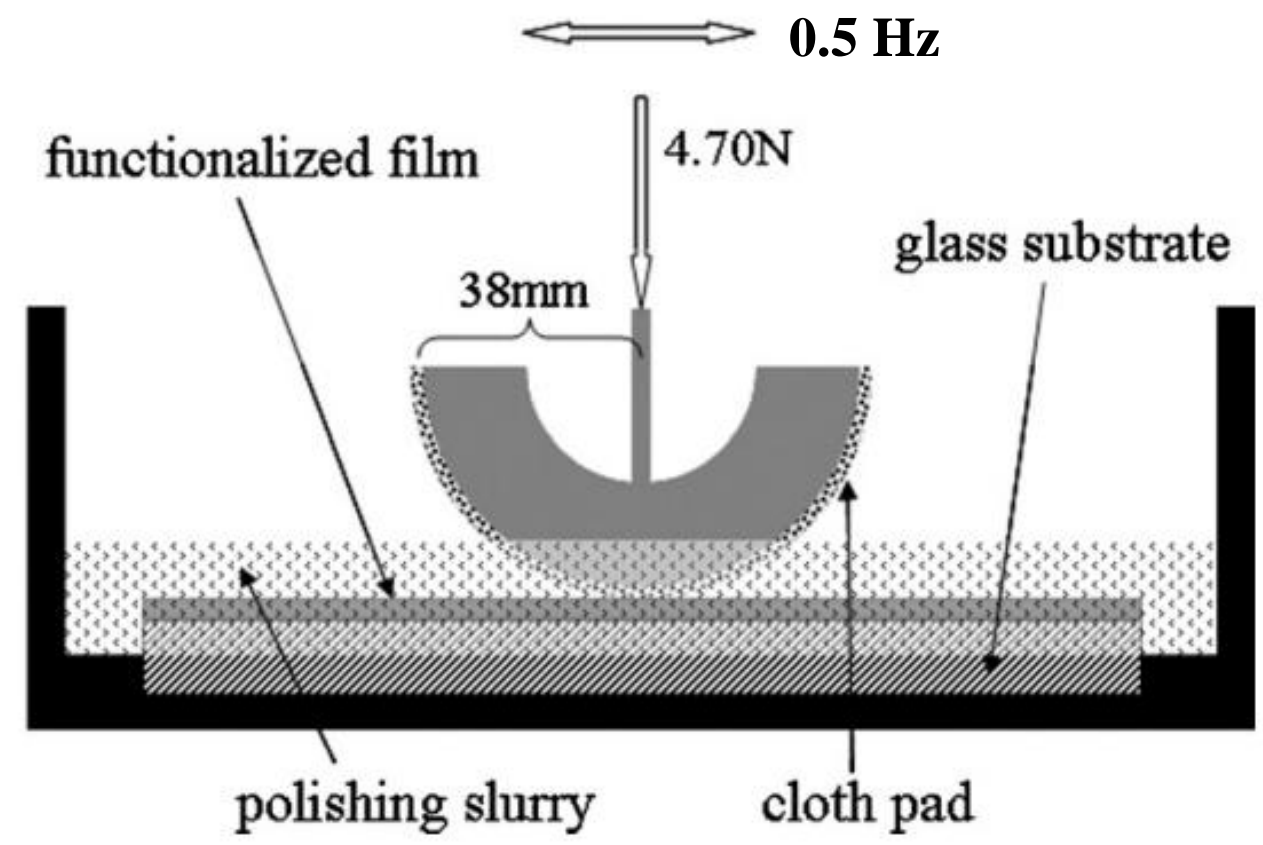

Figure 7: Reciprocating polishing wear apparatus schematic [32]

The wear rate was quantified using two different methods. The first of these methods involves measuring the coating thickness after abrasive wear cycle intervals at a manual scratch as described in Section 3.2.2. Using the Archard equation [33], the coating wear rate can be calculated from measurements of the film thickness after multiple sliding distances (intervals). From the change in thickness, the volume of material removed can be calculated from the change in thickness at each interval. This is done until the coatings are completely worn, and a linear 
least squares regression fit of film thickness versus reciprocating wear cycles is found. The Archard equation used is as follows:

$$
\mathrm{K}=\frac{\mathrm{V}}{\mathrm{S} \times \mathrm{N}}
$$

where $\mathrm{V}$ is the wear volume in $\mathrm{mm}^{3}, \mathrm{~S}$ is the total sliding distance in meters, and $\mathrm{N}$ is the applied normal load in newtons.

The second method of calculating the wear rate of each coating was by measuring the mass loss after intervals of reciprocating cycles until the coating is worn down to the glass [32]. The wear rate was calculated as the rate of mass loss per cycle. A high sensitivity balance (DV215CD, OHAUS Corp., Parsippany, NJ) with resolution of $0.01 \pm 0.02 \mathrm{mg}$ was used to measure the mass loss after each interval. Care was taken to avoid inaccuracies from small amounts of dust or alumina on the glass slides by cleaning with deionized water, an alcohol solution, and allowing the slides to dry for 10 minutes to equilibrate to room conditions [32].

\subsubsection{Linear Reciprocating Tribometer}

In addition to the in-lab built abrasive wear testing, a CSM Instruments MicroTribometer was used to determine the friction coefficient and wear characteristics of select coatings. An image of this tribometer is shown in Figure 8. The samples were mounted in the linear-reciprocating module and reciprocated under the static contact of a stainless steel ball (10 $\mathrm{mm}$ diameter). The normal load applied was $0.75 \mathrm{~N}$ and the stroke length was $10 \mathrm{~mm}$ at $0.16 \mathrm{~Hz}$ with a maximum linear speed of $10 \mathrm{~mm} / \mathrm{s}$ for 100 cycles for each run. The steel ball was rotated 
after each use to give a pristine surface for each new measurement. All measurements were done in ambient room conditions.

The output of the Micro-Tribometer includes a graph of the penetration depth into the coating as well as the coefficient of friction as calculated simply as the ratio of the measured tangential force on the tribometer arm and the normal force of $0.75 \mathrm{~N}$ applied.

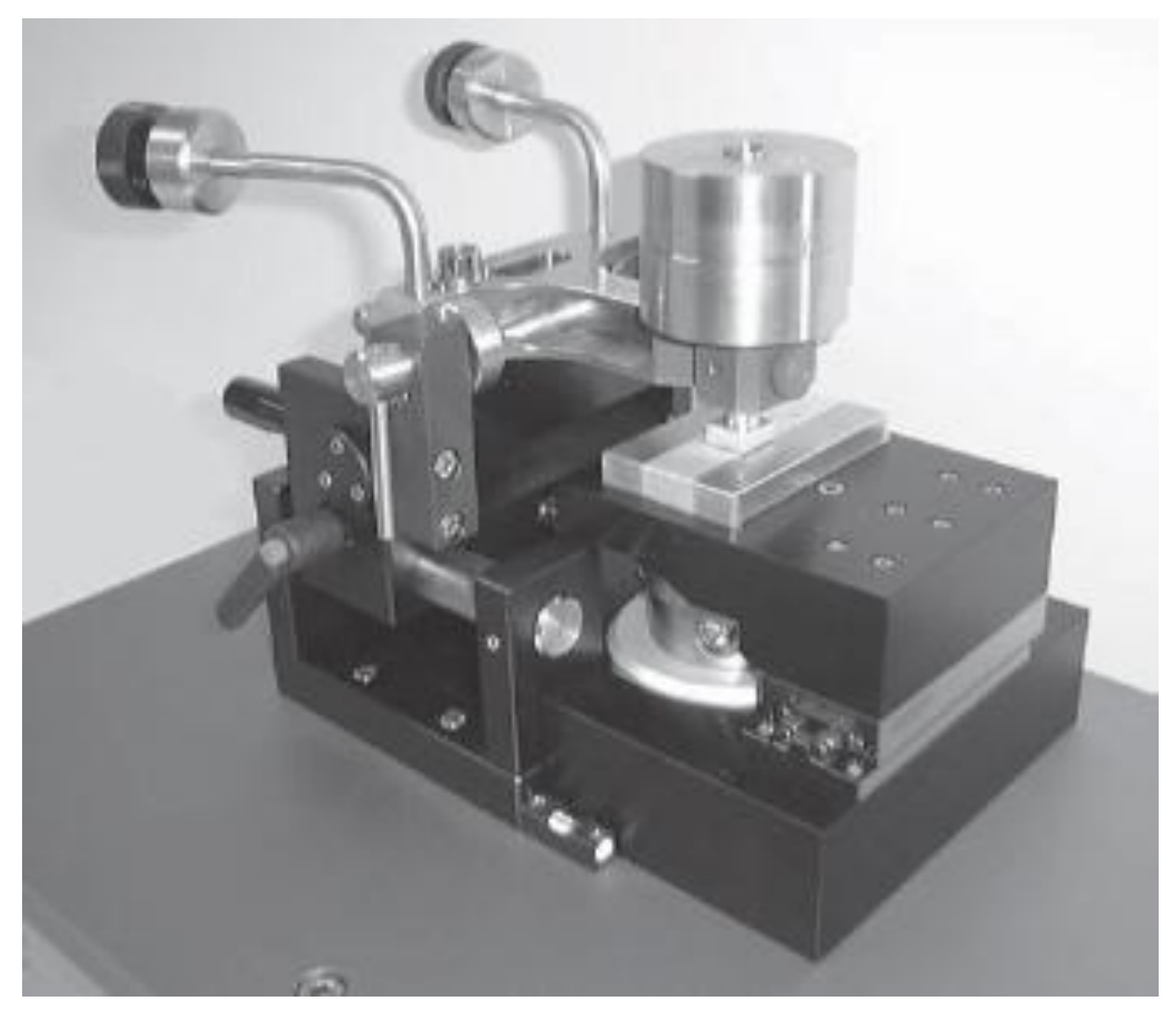

Figure 8: Linear-reciprocating Micro-Tribometer (CSM Instruments)

\subsubsection{Contact Angle Goniometry}

Before and after intervals of abrasion with the lab-built reciprocating wear apparatus, the hydrophobicity of each coating was analyzed. This hydrophobicity was quantified using a lab- 
built sessile drop contact angle goniometer $[5,7,10,11,32]$. Five approximately $5-\mu \mathrm{L}$ droplets of deionized water were placed over a macroscopic area of the coating approximately 1-2 mm apart using a manual microsyringe. Images of the horizontal view of the drops were captured using a digital microscope and analyzed using the low-bond axisymmetric drop shape analysis (LBADSA) method in the "Drop Shape Analysis" plugin [34] for ImageJ.

\subsubsection{Nanoindentation}

The hardness (H) and elastic modulus (E) of each coating were measured using instrumented indentation testing, as developed by Oliver and Pharr [35]. Instrumented indentation is the most appropriate measurement method for thin coatings and soft materials. This established method involves an indenter tip with a known geometry being driven into a specific site of a material to be tested by applying an increasing normal load. When reaching a pre-set maximum value, the normal load is reduced until partial or complete relaxation occurs. The resulting load/displacement curves provide data specific to the mechanical nature of the material. This eliminates the need for analyzing the image of a residual indent in the material, which generally leads to false results in thin films. Thus, instrumented indentation accounts for both the plastic and elastic deformation, as shown in Figure 9. 


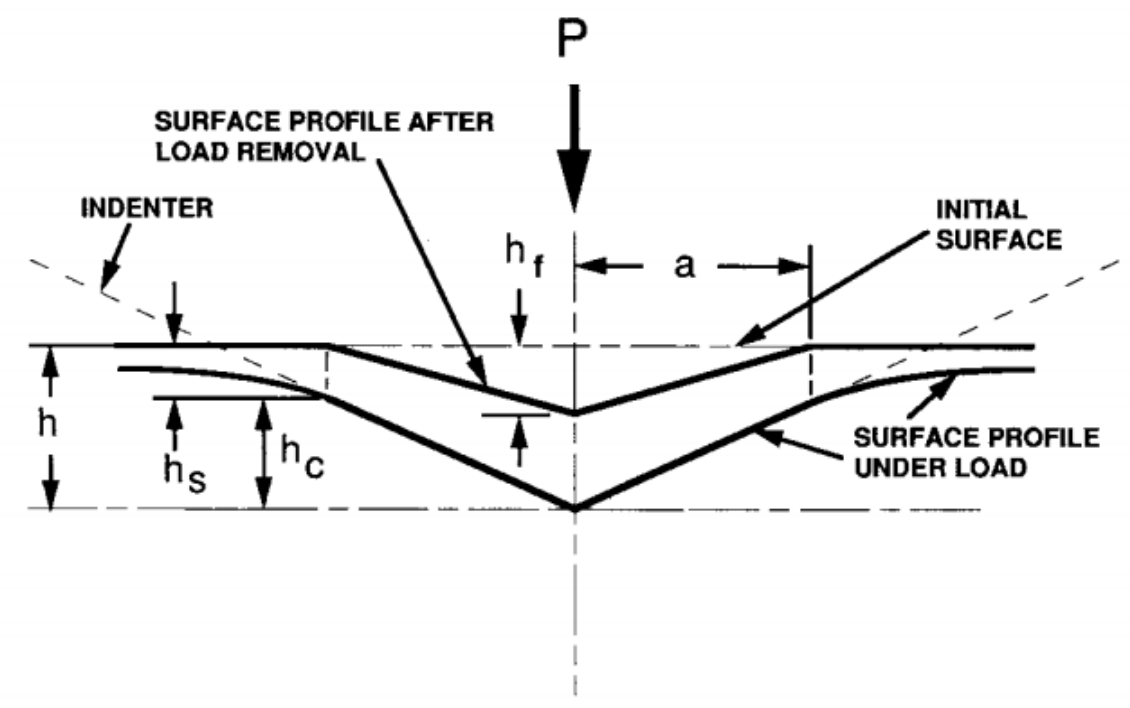

Figure 9: Schematic of a cross-section through an indentation [36]

Through a series of mathematical equations, the instrumented hardness $\left(\mathrm{H}_{\mathrm{IT}}\right)$ and instrumented elastic modulus $\left(\mathrm{E}_{\mathrm{IT}}\right)$ are calculated. The instrumented hardness is expressed by the ratio between the applied load and the contact area, expressed as:

$$
\mathrm{H}_{\mathrm{IT}}=\frac{\mathrm{F}_{\max }}{\mathrm{A}_{\mathrm{p}}}
$$

where $F_{\max }$ is the maximum load and $A_{p}$ is the contact area between the indenter and the specimen at the maximum depth and load. $\mathrm{F}_{\max }$ is known from the software, and $\mathrm{A}_{\mathrm{P}}$ is calculated from a known reference material.

A reduced modulus, $\mathrm{E}_{\mathrm{IT}}{ }^{*}$, is used to account for the fact that the elastic displacements occur in both the indenter and the sample. The instrumented elastic modulus in the test material, $\mathrm{E}_{\mathrm{TT}}$, is calculated from $\mathrm{E}_{\mathrm{IT}} *$ using the following: 


$$
\frac{1}{E_{I T}^{*}}=\frac{\left(1-v^{2}\right)}{E_{I T}}+\frac{\left(1-v_{i}^{2}\right)}{E_{i}}
$$

where $v$ is the Poisson's ratio for the sample and $E_{\mathrm{i}}$ and $v_{\mathrm{i}}$ are the elastic modulus and Poisson's ratio, respectively, of the indenter tip. Furthermore, the reduced elastic modulus is linked to the measured stiffness, S, from the unloading curve of the load-displacement graph of an indent by the relation:

$$
\mathrm{E}_{\mathrm{IT}}^{*}=\frac{\sqrt{\pi}}{2} \frac{\mathrm{S}}{\sqrt{\mathrm{A}_{\mathrm{p}}}}
$$

Graphically, this stiffness parameter $S$ corresponds to the tangent of this unloading curve, as shown in Figure 10.

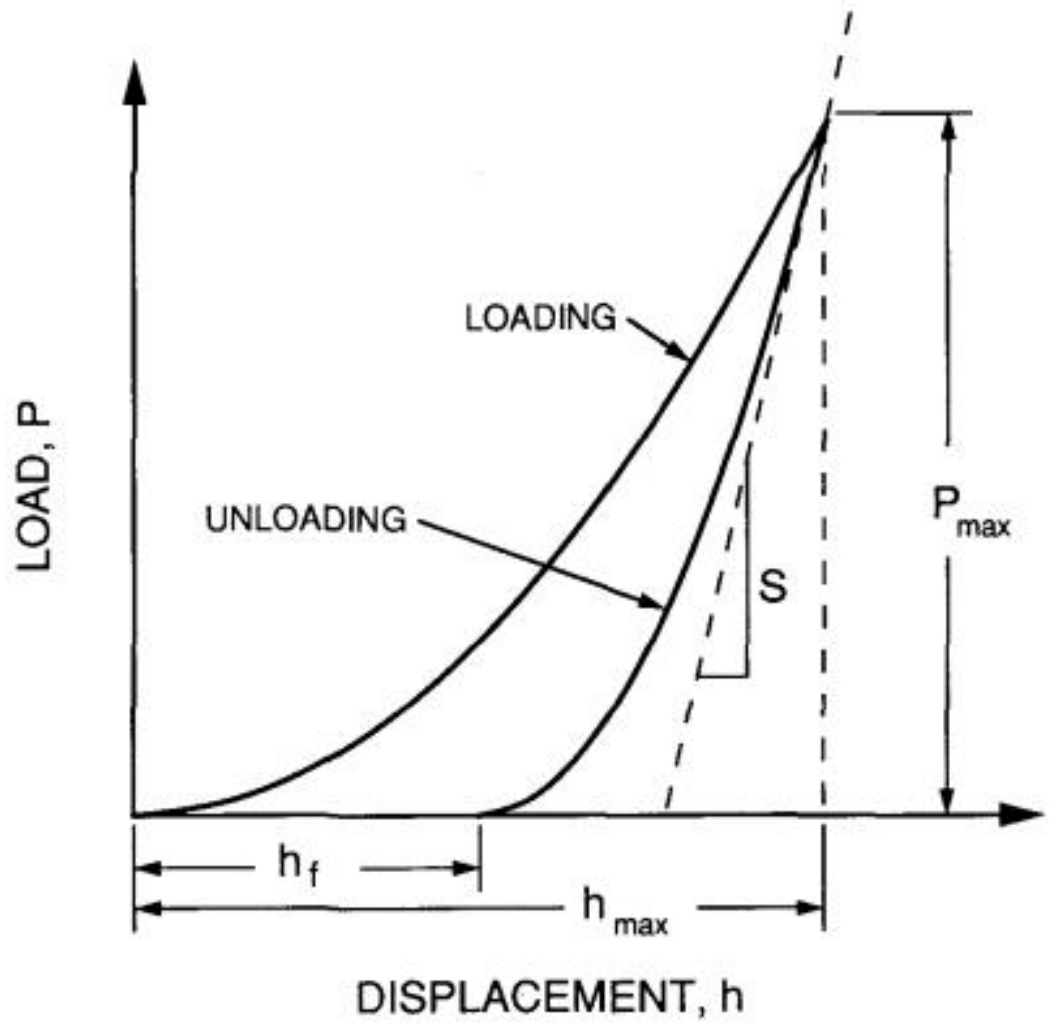

Figure 10: Schematic of load vs. displacement curve for an indent [36] 
With the values of $S$ and $A_{p}$, $E_{I T} *$ can be calculated. $E_{I T}$ is thus determined from Equations (4) and (5) by the equation:

$$
\mathrm{E}_{\mathrm{IT}}=\frac{\left(1-v^{2}\right)}{\frac{1}{\mathrm{E}_{\mathrm{IT}}^{*}}-\frac{\left(1-v_{\mathrm{i}}^{2}\right)}{\mathrm{E}_{\mathrm{i}}}}
$$

For the case of a relatively soft coating on a hard substrate, it is especially desirable to use low normal loads and achieve low penetration depths into the surface to avoid substrate effects on the analysis. It is generally accepted that the maximum penetration depth into the coating should be less than $10 \%$ of the coating thickness to avoid these substrate effects. Similarly, the International Standard ISO 14577-4 stipulates that the average roughness $\left(\mathrm{R}_{\mathrm{a}}\right)$ should be less than $5 \%$ of the maximum penetration depth.

It is thus imperative to know the condition of a surface before proceeding with an instrumented indentation test. The lowest maximum loads possible should be used that provide reliable and repeatable data. CSM Instruments' NHT Nanoindentation Tester and UNHT Ultra Nanoindentation Tester were used for these analyses. The NHT and UNHT specifications are listed in Table 3. Also, a basic schematic of the NHT indentation system is shown in Figure 11.

Table 3: Specifications of the CSM Instruments' NHT and UNHT

\begin{tabular}{|c|c|c|}
\hline & NHT & UNHT \\
\hline Load Range & $0.1-500 \mathrm{mN}$ & $0.020-100 \mathrm{mN}$ \\
\hline Load Resolution & $0.04 \mu \mathrm{N}$ & $0.001 \mu \mathrm{N}$ \\
\hline Depth Resolution & $0.04 \mathrm{~nm}$ & $0.001 \mathrm{~nm}$ \\
\hline
\end{tabular}




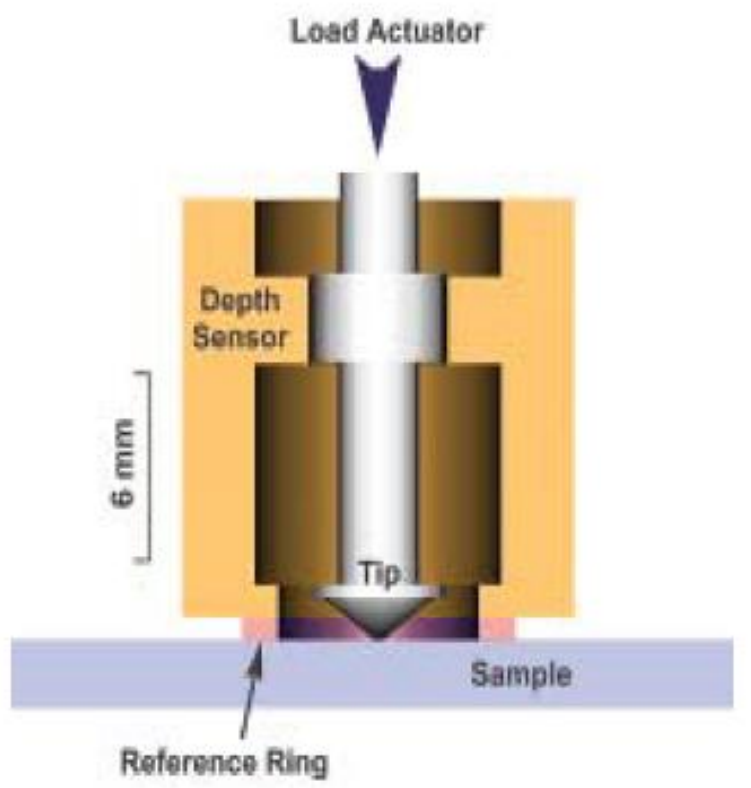

\section{Figure 11: Schematic of NHT indentation system [36]}

The UNHT provides the best and most reliable results for this case of soft coatings on hard substrates. The instrument is equipped with a patented double referencing system that allows for more reliably applied low normal loads and higher sensitivity. The lower load range allows the instrument to work more comfortably within its limits for these sol-gel coatings compared to the NHT. The parameters used with the UNHT were constant for all samples, with an approach speed of $700 \mathrm{~nm} / \mathrm{min}$, a maximum load of $0.10 \mathrm{mN}$, a loading and unloading rate of $0.20 \mathrm{mN} / \mathrm{min}$, and a pause of 10 seconds. The standard indentation method was used with a 30 second load, followed by the specified pause, and finally a 30 second unload. The only indenter used with the UNHT was the Berkovich diamond tip. For all coatings, the penetration depth was kept below $10 \%$ of the coating thicknesses with the UNHT. All measurements were conducted in ambient air $\left(23 \pm 1{ }^{\circ} \mathrm{C}, 40 \pm 10 \%\right.$ relative humidity), and fused silica is used as the reference material for the indenter tip. Figure 12 shows the sample setup under the UNHT. 


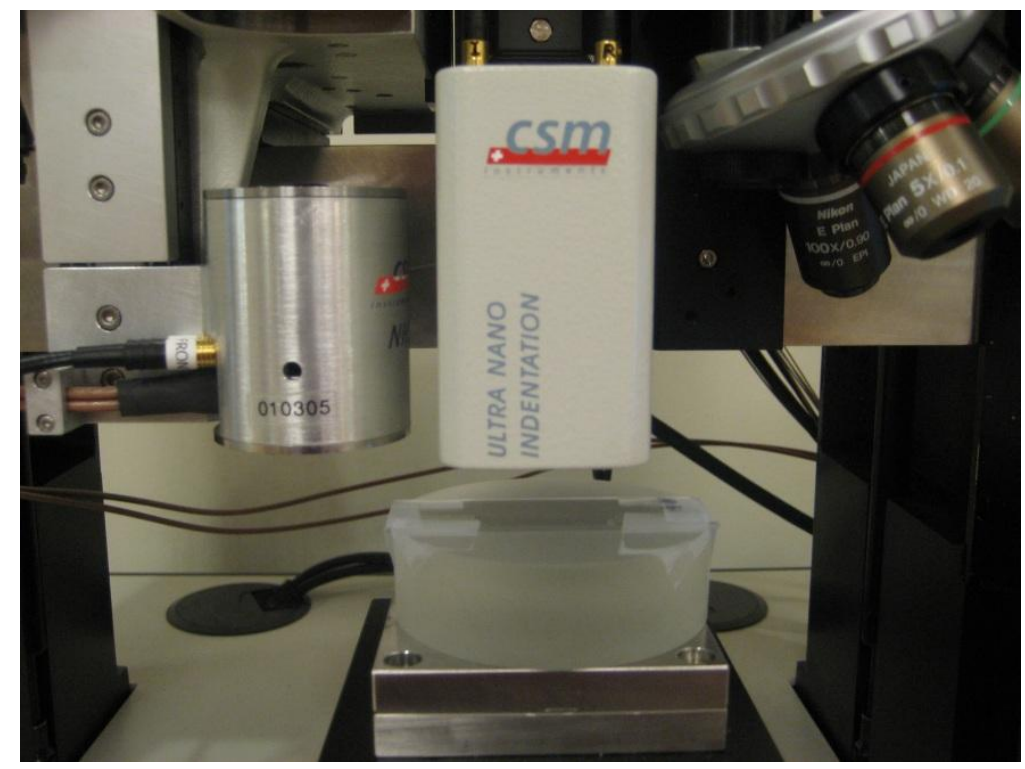

Figure 12: Sample setup under UNHT Ultra Nanoindentation Tester

The NHT was used similarly to the UNHT but with a slightly higher load and penetration depth for the standard indent protocol. Maximum normal loads used were between 0.4 and 1.0 $\mathrm{mN}$, with loading rates such that the loading and unloading times were 30 seconds each. The NHT was used to do a large number of indents in a short period of time to determine a statistical distribution of hardness and modulus data throughout the coatings. For the NHT, both a Berkovich diamond tip indenter and a $10 \mu \mathrm{m}$ radius sphero-conical diamond tip indenter were used. Fused silica is also used as the reference material for the both of these indenter tips. Figure 13 shows the sample setup under the NHT.

In addition to this standard indent protocol, a continuous multicycle (CMC) protocol was used for analysis with the NHT system. This CMC protocol is similar to the standard protocol until the unloading portion. While unloading, the indenter retracts to only $50 \%$ of the maximum load and then increases to a maximum load higher than the previous cycle. This is repeated a specified number of times to a specified maximum load. This technique provides some insight 
into the hardness and elastic modulus at different depths at the same position of a material. This can be especially helpful when dealing with sol-gel coatings with complex structures and surface-segregating molecules.

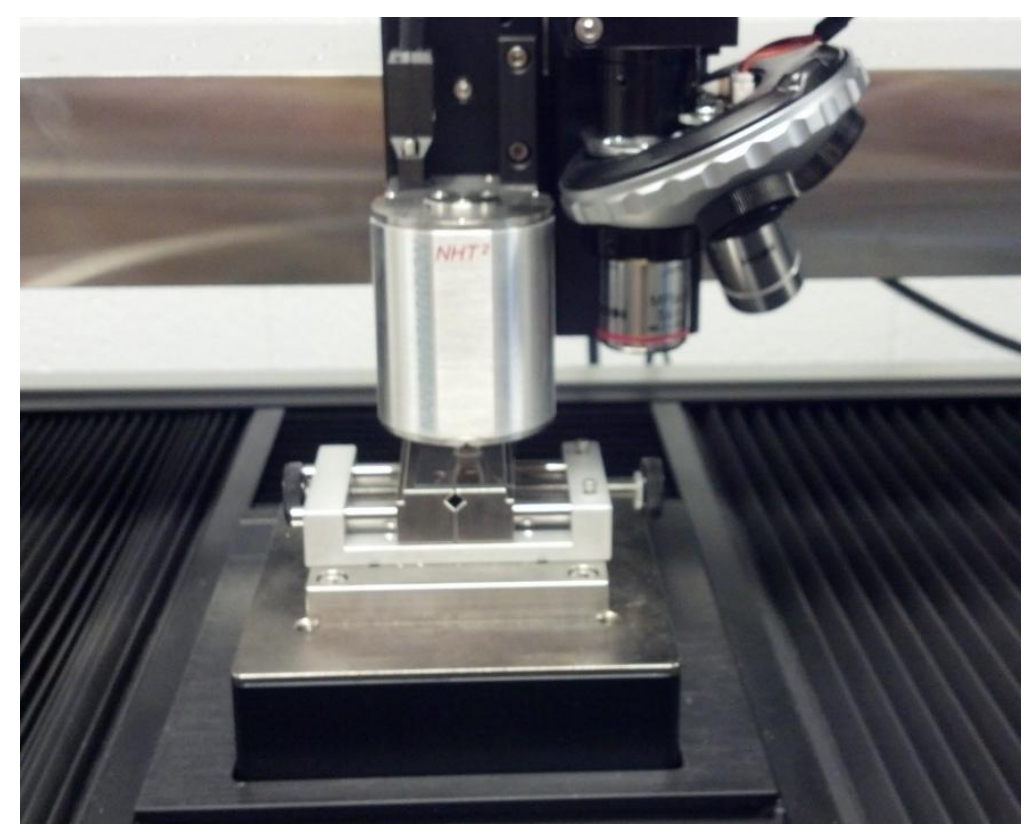

Figure 13: Sample setup under NHT Nanoindentation Tester

\subsubsection{Scratch Testing}

To determine the coating adhesion and the normal load at which critical failure of the coating occurs, scratch testing was performed using a Nano-Scratch Tester (NST, CSM Instruments, Switzerland). The advantage of automated low-load scratch testing is its ability to analyze the friction force and coefficient at a measured penetration depth and normal force. This can then be correlated to the image of the scratch itself to better detect failure mechanisms. This can be especially useful for composite coatings as there may be multiple methods of failure in the coating other than just adhesive failure. 
Samples were mounted on a standard resolution friction table (ST-142, $0.01 \mathrm{mN}$ friction force resolution) and scratched with a $10 \mu \mathrm{m}$ radius Rockwell diamond tip indenter. A progressively increasing load scratch program was used from $5 \mathrm{mN}$ to $75 \mathrm{mN}$ over a length of $500 \mathrm{um}$, with a loading rate of $140 \mathrm{mN} / \mathrm{min}$. All measurements were conducted in ambient room temperature conditions. Figure 14 shows the sample setup under the NST.

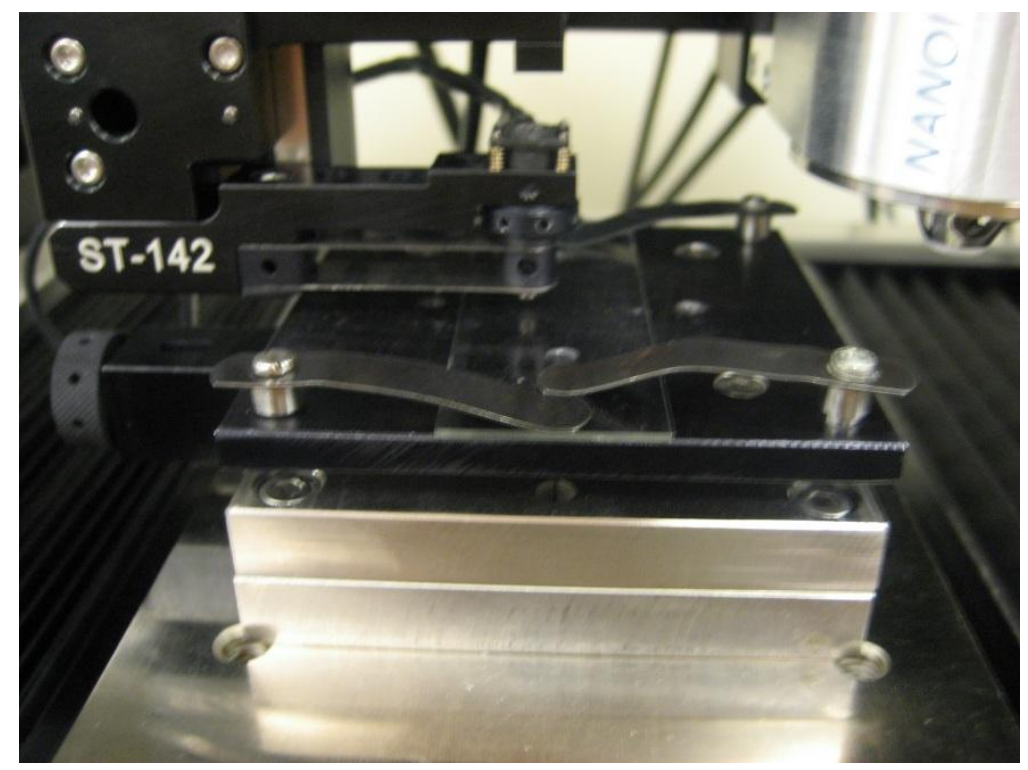

Figure 14: Sample setup under Nano-scratch Tester 


\section{Results and Discussion}

\subsection{Optical and Structural Analysis}

\subsubsection{Optical Transmission}

Optical transmission measurements showed that the coatings with added colloidal silica up to $2 \%$ had as good or better transparency than the clean soda-lime glass slides on the visible spectrum. Differences in the coatings arise from their varying compositions and microstructures. The roughness $R_{a}$ as measured by stylus profilometry of each pristine coating was below $5 \mathrm{~nm}$ until after a loading of about $3 \%$ added colloidal silica to the non-templated coatings. At this point, $\mathrm{R}_{\mathrm{a}}$ values increased to between $10-15 \mathrm{~nm}$, with no discernible difference in the $5 \%$ and $10 \%$ added silica coatings.

The glass slide transparency was found to be an average of $90.5 \%$ across the visible range, as shown in Figure 15, Figure 16, Figure 17, and Figure 18 for comparison purposes. Figure 15 shows the optical transmission of a non-templated coating with no added silica. This slide shows a higher average transmission and interference fringes indicative of coatings with a lower refractive index than the substrate. This has been shown previously to result from mesopores and embedded fluorinated organics [7]. Figure 16 shows an even higher average transmission with $1 \%$ added colloidal silica, while at $2 \%$ in Figure 17 there is a slight decrease in transmission on the lower end of the visible spectrum. At a colloidal silica concentration of 5\% there is an even more well-defined drop in transparency, as shown in Figure 18. 


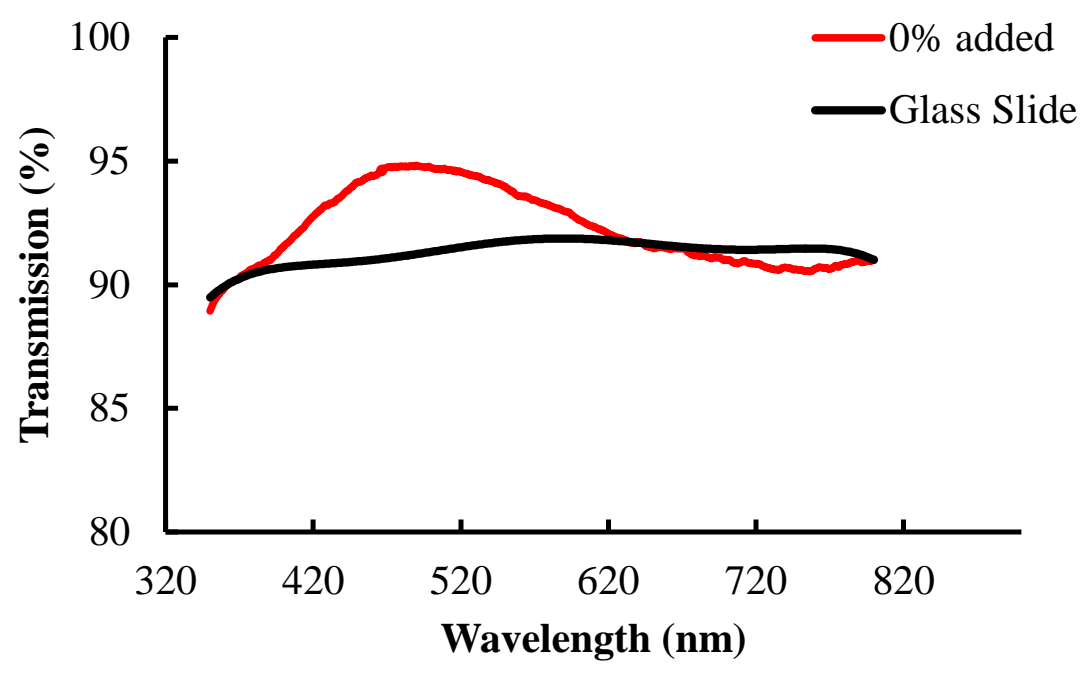

Figure 15: Optical transmission spectrum for non-templated coating with no added silica

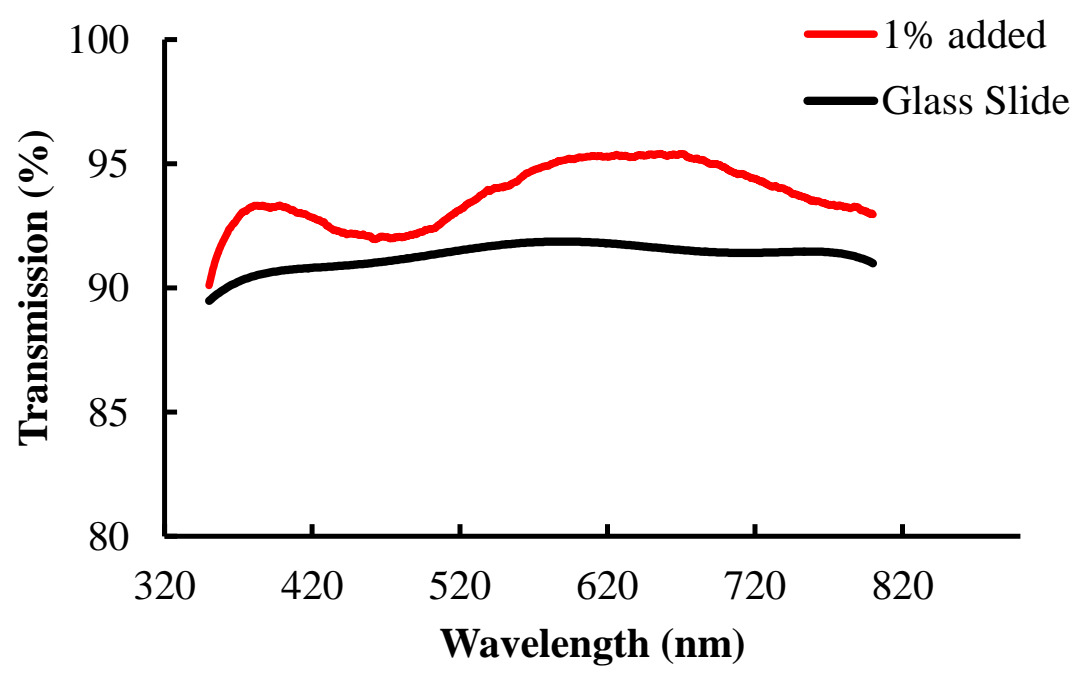

Figure 16: Optical transmission spectrum for non-templated coating with 1\% added silica 


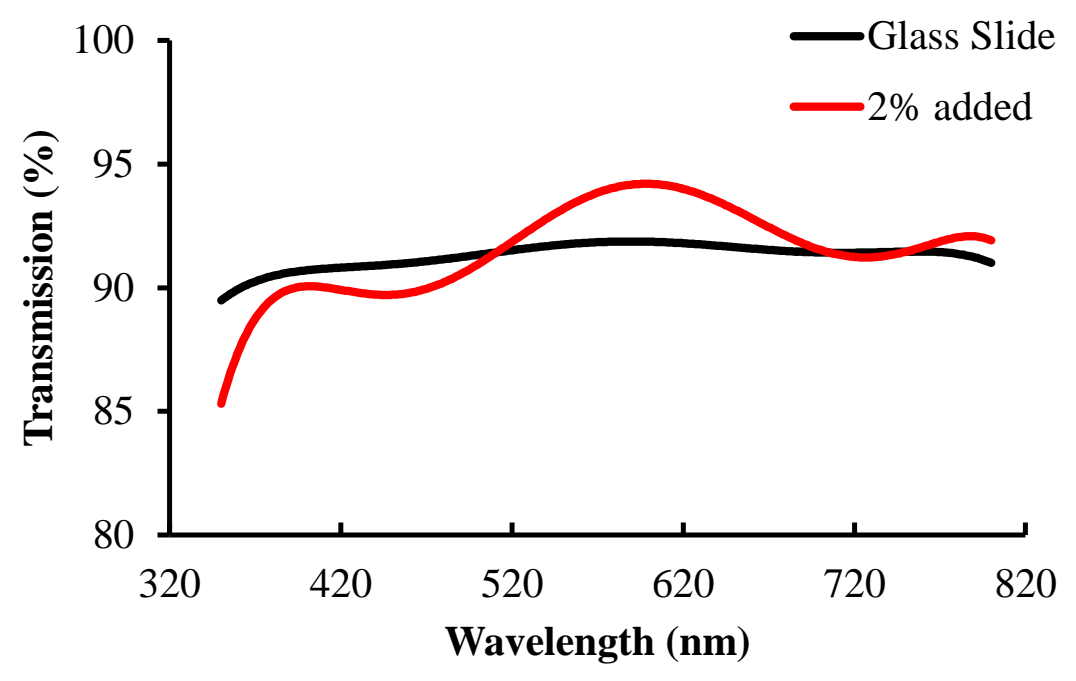

Figure 17: Optical transmission spectrum for non-templated coating with $2 \%$ added silica

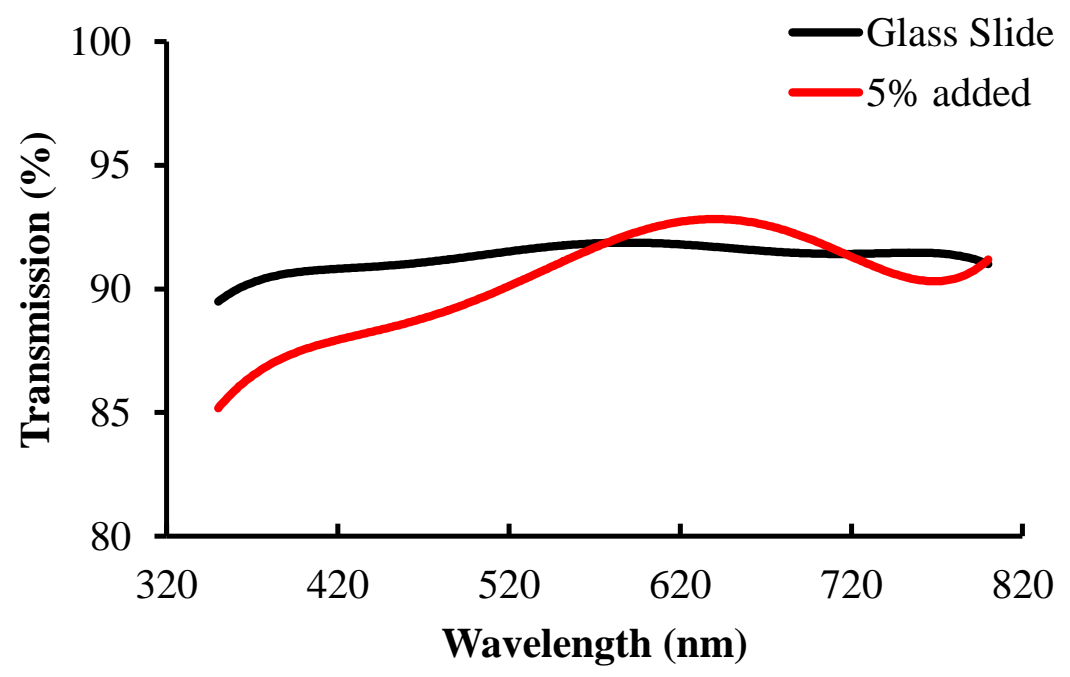

Figure 18: Optical transmission spectrum for non-templated coating with $\mathbf{5 \%}$ added silica 
To further elucidate this issue of loss of transparency, optical microscopy of each of these coatings was performed. As shown in Figure 19, there is a clearly visible agglomeration of particles on the sample surface. Above the $2 \%$ concentration in Figure 19(d), the surface appears to be densely packed with particles, likely contributing to the noticeable drop in optical transmission.
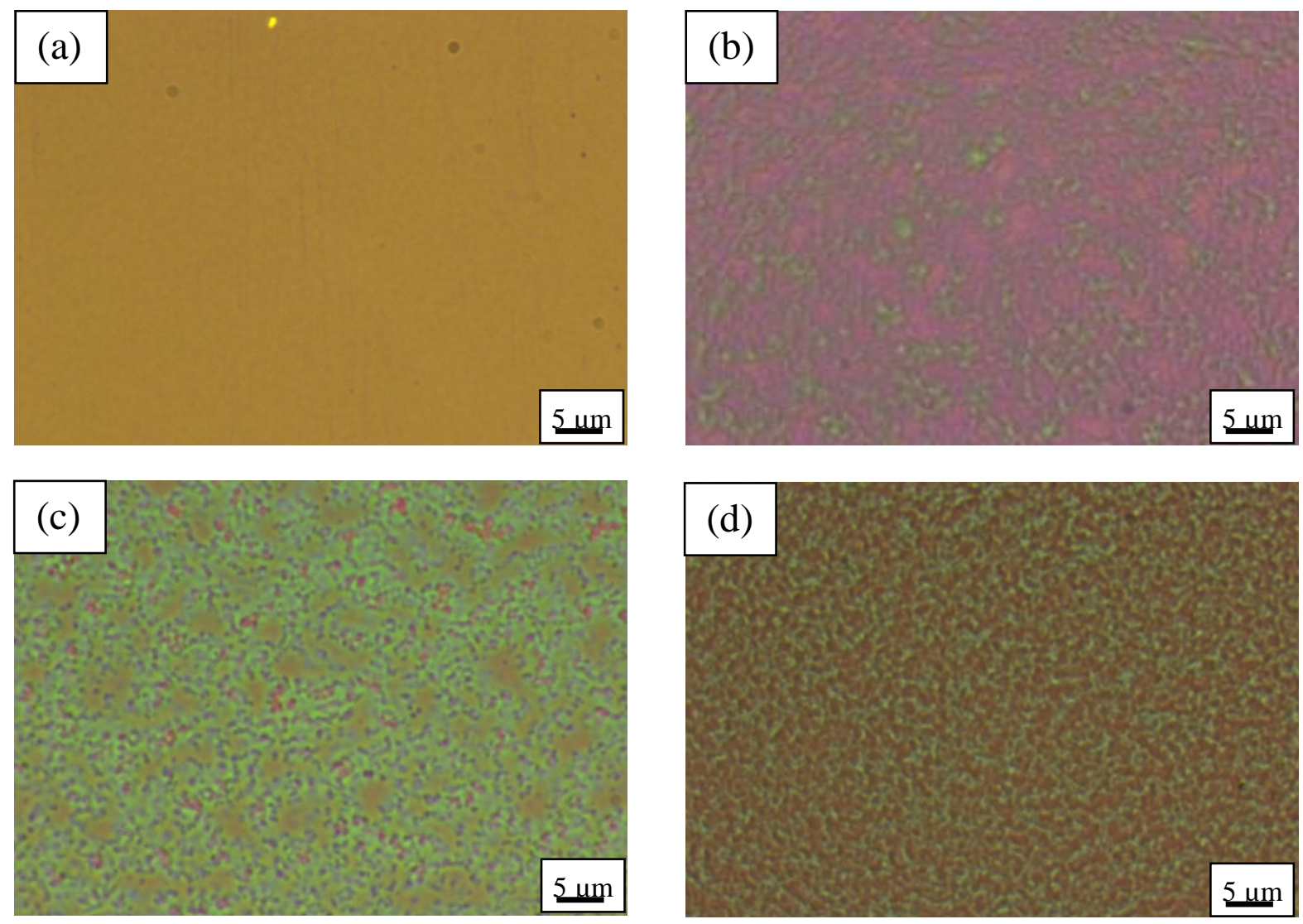

Figure 19: Optical micrographs of non-templated coatings with (a) $0 \%$, (b) $1 \%$, (c) $2 \%$, and (d) $5 \%$ added colloidal silica 


\subsubsection{Atomic Force Microscopy}

Atomic force microscopy tapping mode was used to produce topographical maps of the added colloidal silica surfaces. Figure 20 shows a $10 \mu \mathrm{m}$ by $10 \mu \mathrm{m}$ tapping mode topograph for a non-templated coating with $2 \%$ added colloidal silica. This scan was performed at a scan speed of 0.996 lines/s or $19.93 \mu \mathrm{m} / \mathrm{s}$. The frequency was set to $388.6 \mathrm{kHz}$ and the force setpoint was 4.507 $\mathrm{V}$ for this scan. Figure 21 shows the cross section of this topograph at the green line on Figure 20. From these scans, it was determined that the average roughness for this coating was $6.8 \mathrm{~nm}$.

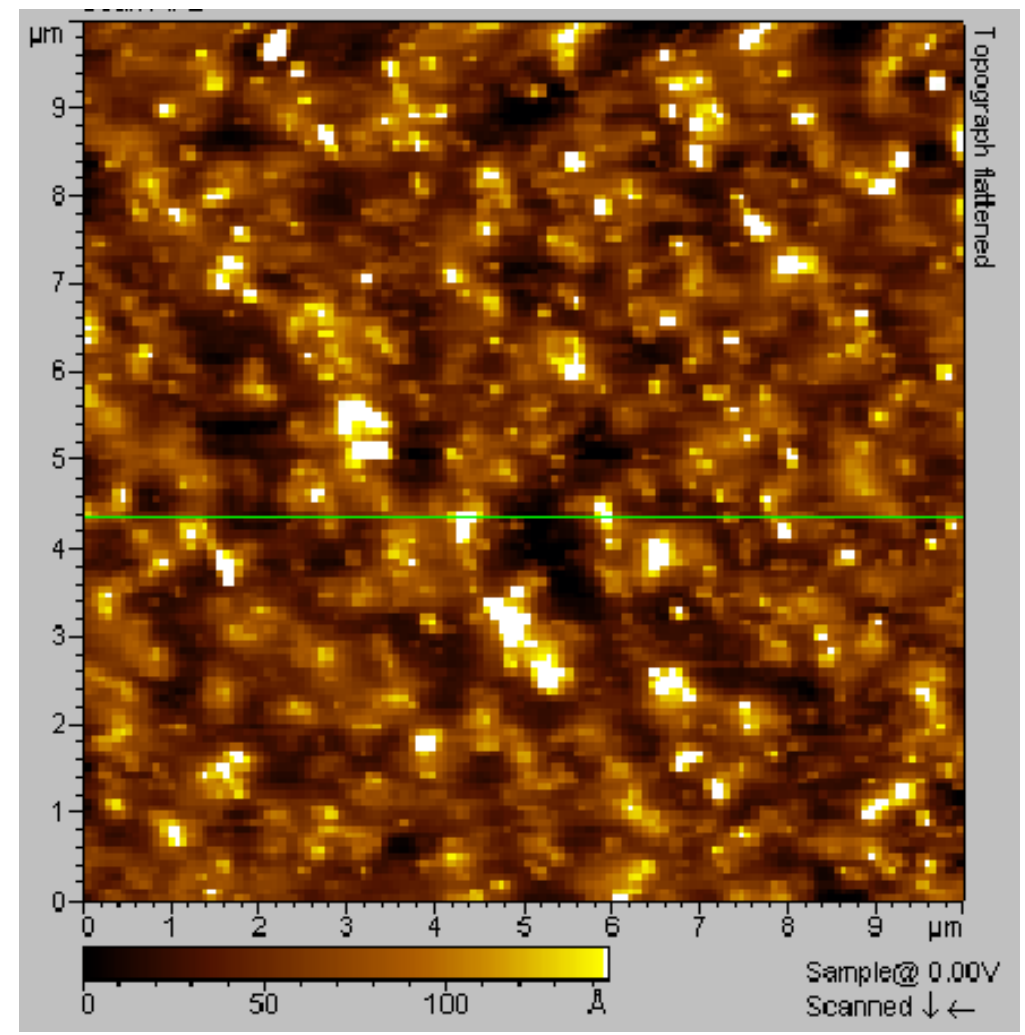

Figure 20: AFM tapping mode micrograph of a non-templated coating with $2 \%$ added colloidal silica 


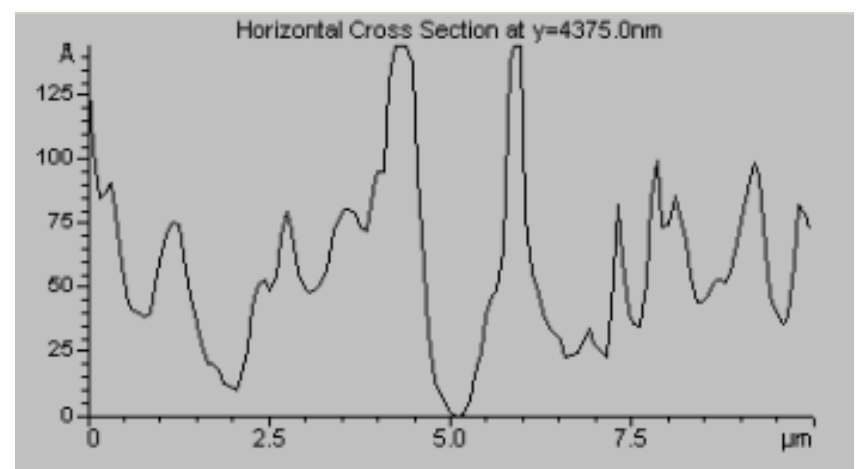

Figure 21: Topography cross section using AFM through a non-templated coating with 2\% added colloidal silica corresponding to the green line from Figure 20.

Figure 22 shows a similar topography map but with $3 \%$ added colloidal silica. The surface mapping and a representative profile of this coating (Figure 23), show a much rougher roughness compared to that of the $2 \%$ added colloidal silica. This can be attributed to a larger particle size on the surface due to agglomeration of the silica nanoparticles in the sol and surface segregation. The average roughness of this coating was found to be $18.6 \mathrm{~nm}$. 


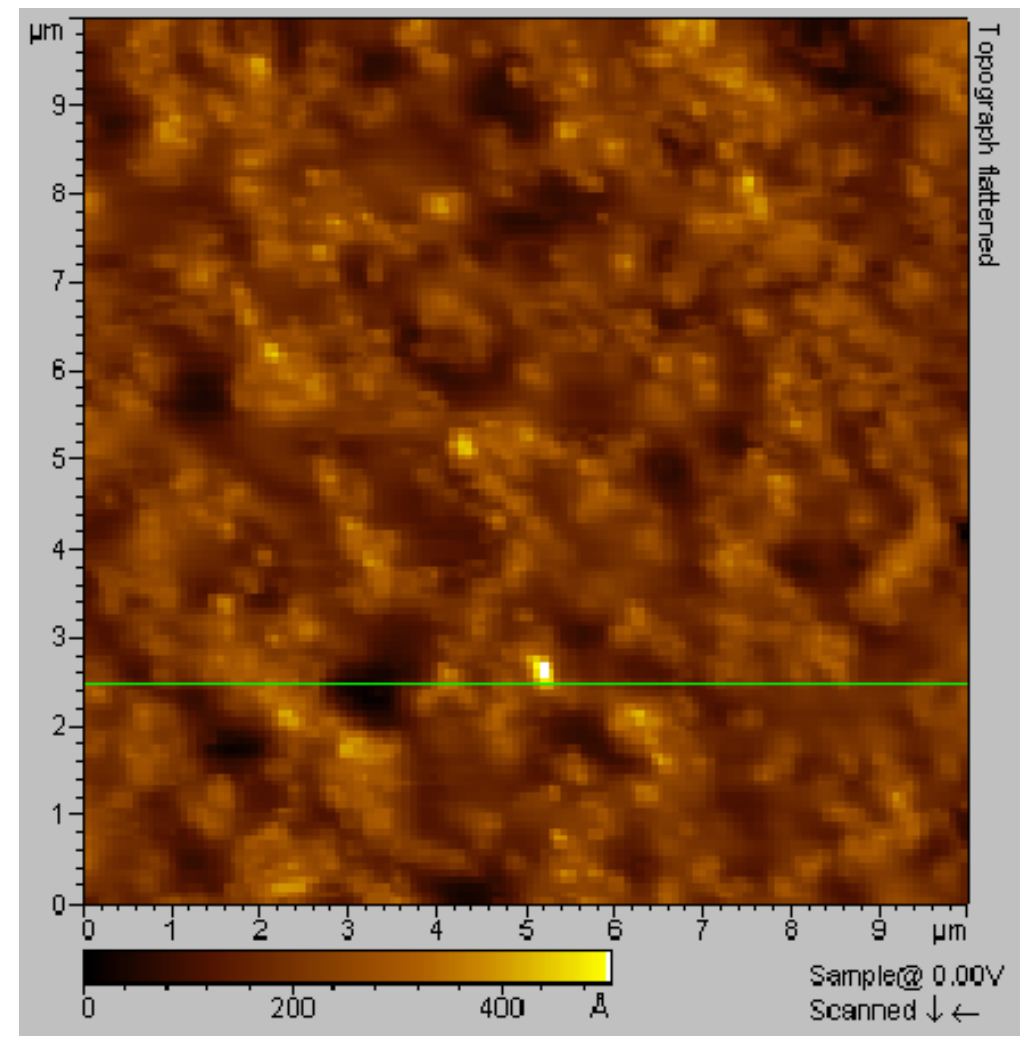

Figure 22: AFM tapping mode micrograph of a non-templated coating with $3 \%$ added colloidal silica.

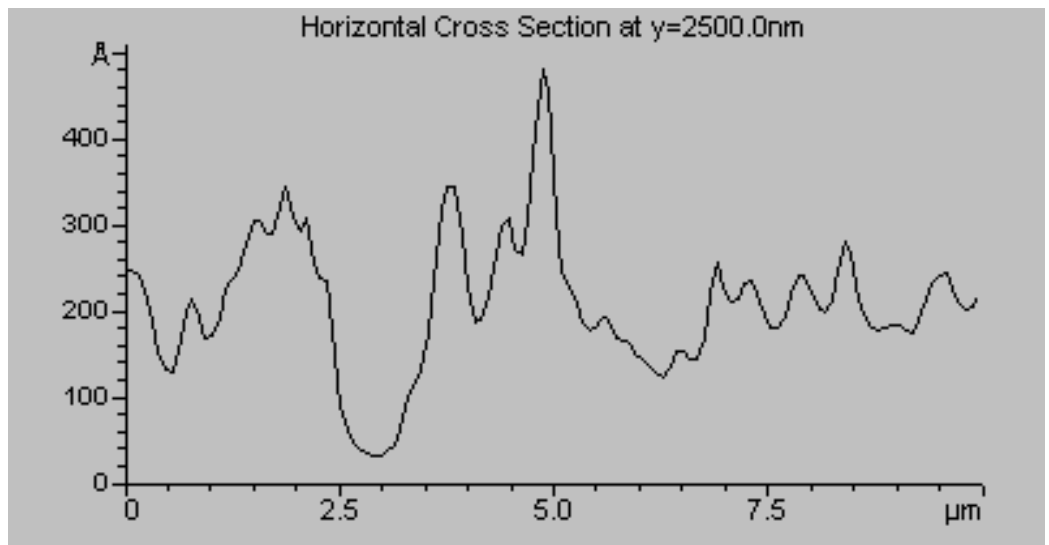

Figure 23: Topography cross section using AFM through a non-templated coating with $3 \%$ added colloidal silica corresponding to the green line from Figure 22.

AFM contact mode was also used to produce topographical maps of the added colloidal silica surfaces. Figure 24(a) shows the topographical map of a templated coating with $2 \%$ added 
colloidal silica. Figure 25 shows the cross-section corresponding to the green line on Figure 24. This scan was $2 \mu \mathrm{m}$ by $2 \mu \mathrm{m}$, with a scan speed of 1.27 lines/s or $5086 \mathrm{~nm} / \mathrm{s}$, and an applied voltage of $0.1 \mathrm{~V}$. The average roughness for this coating was found to be $20.2 \mathrm{~nm}$. Thus, there is an increase in roughness with the addition of a surfactant template similar to that of adding $1 \%$ more colloidal silica.

Figure 24(b) shows a friction force mapping of this coating. This technique provides a high resolution image of a surface with contact mode and also allows for further insight into the correlation between friction and wear of a specific coating [10].

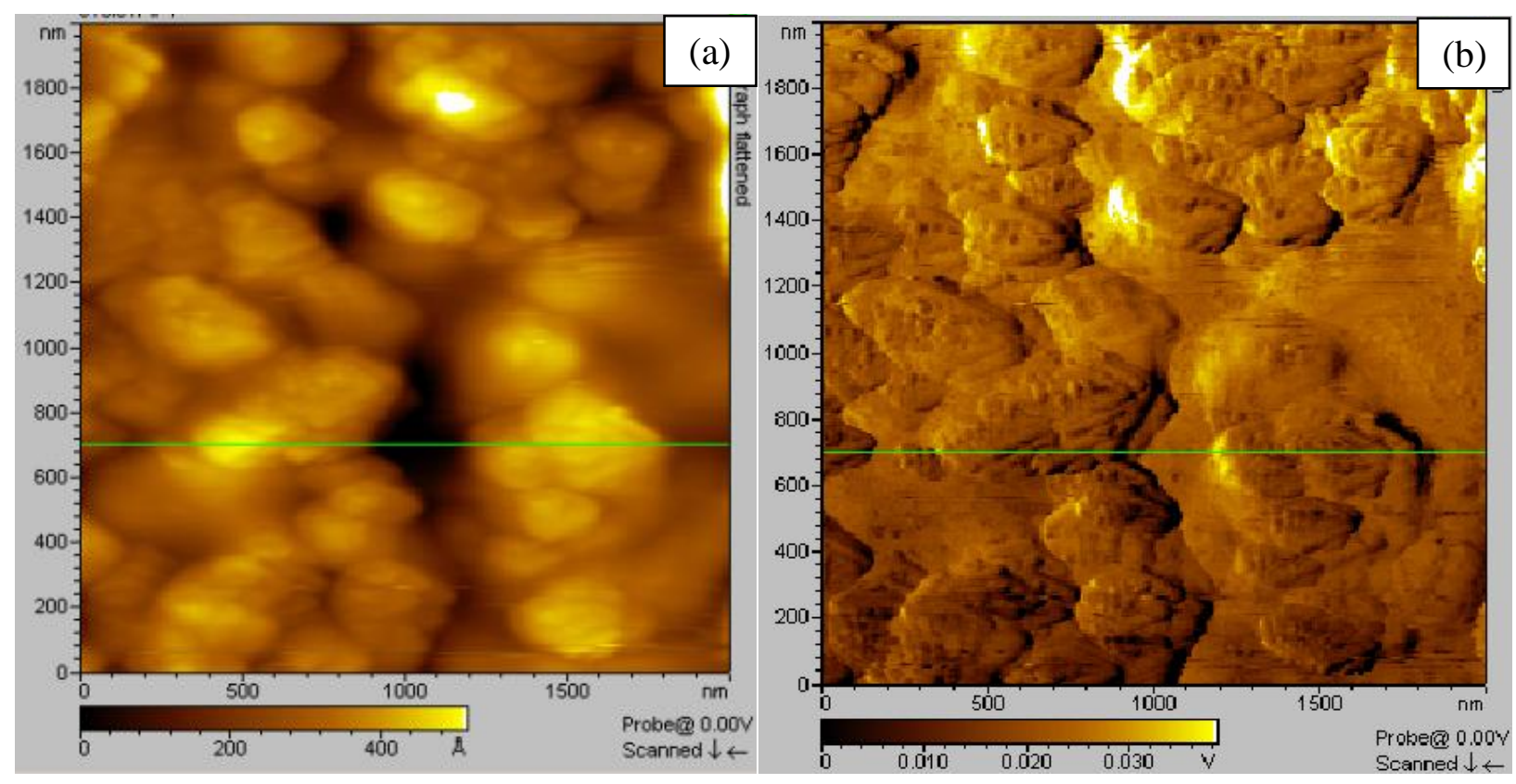

Figure 24: AFM contact mode micrographs of a templated coating with $2 \%$ added colloidal silica. (a) Topographical mapping and (b) friction force mapping. 


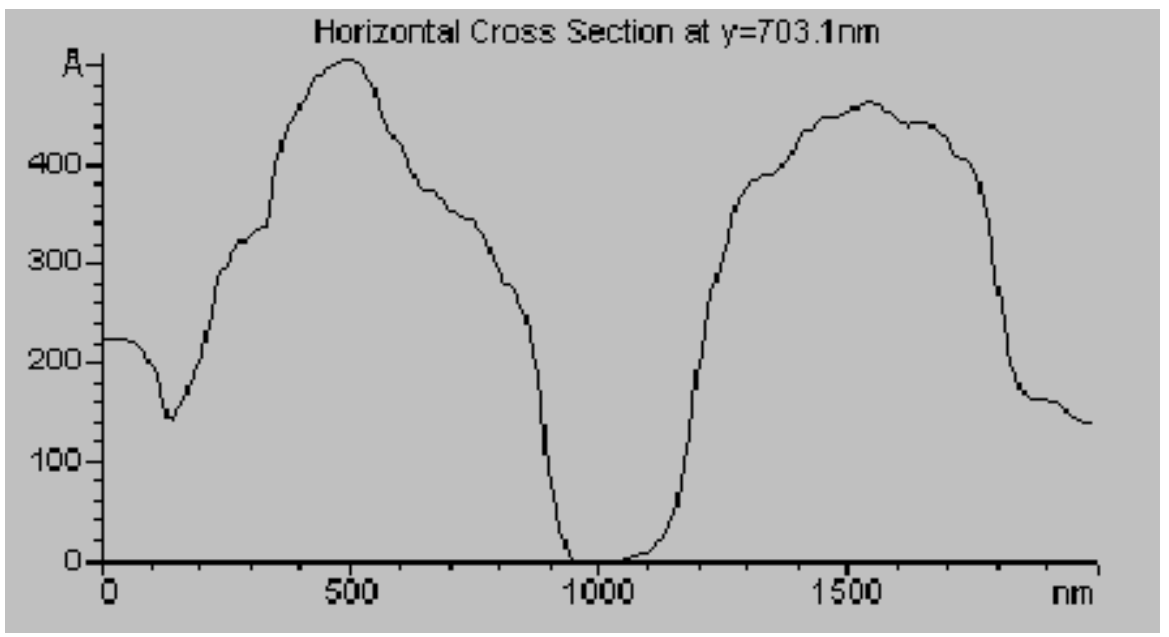

Figure 25: Topography cross section using AFM through a templated coating with 2\% added colloidal silica corresponding to the green line from Figure 24. 


\subsection{Functional Analysis}

The functional analysis of the coatings was analyzed before and after controlled abrasive wear testing. Figure 26 shows examples of droplets used to measure the contact angles of water on the coatings. Figure 26(a) shows the droplets on a pristine surface before wear. After intervals of wear cycles, the hydrophobicity of the coatings is decreased as the bulk of the coating is exposed. This is evident by the decreased contact angle shown in Figure 26(b). After the entire coating is worn and the glass substrate is exposed, there is an even smaller water contact angle, as shown in Figure 26(c).

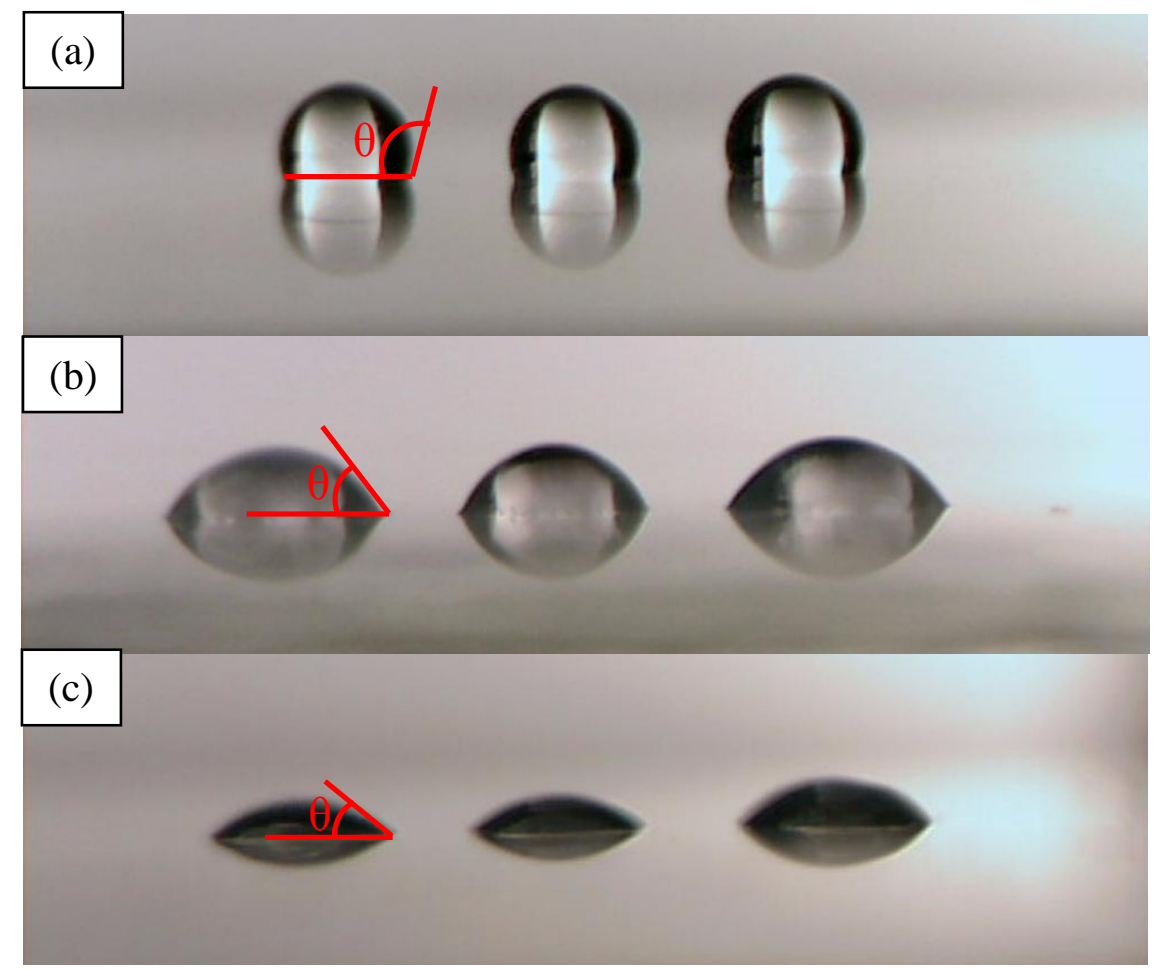

Figure 26: Examples of water droplet contact angles on (a) a pristine surface, (b) a partially worn surface, and (c) bare glass. 
The functional sustainability after intervals of abrasive wear cycles for each nontemplated coating with added colloidal silica is shown in Figure 27. The coatings tested had 0, $0.5,1,2,3,5$, and 10 percent added colloidal silica. For all coatings tested except the $10 \%$ added silica, this initial contact angle was above $95^{\circ}$. The $10 \%$ added colloidal silica had an initial contact angle consistently of $85^{\circ}$. After any initial interval of wear, these contact angles would decrease by between $10^{\circ}$ and $30^{\circ}$. However, the contact angles would not decrease significantly after that point as shown by the nearly flat curves after $20 \%$ penetration depth into the coating. Similarly to work by Kessman et al. [5,7], there is a distinct decline in contact angle and thus hydrophobicity for coatings with no surfactant and no added colloidal silica, shown as $0 \%$ in Figure 27. However, as colloidal silica is added to reinforce the matrix, there is an apparent effect on the functional sustainability of the coatings. This change can possibly be attributed to better dispersion or encapsulation of the fluorosilanes into the bulk of the coating due to the added colloidal silica. While this phenomenon may be a product of multiple factors, it has been noted previously [5] that there is a noticeable induced porosity from added organic molecules within a sol-gel matrix, causing the fluorosilanes to migrate to this solid-vapor interface and thus increasing the bulk hydrophobicity.

Additionally, changing the concentration of colloidal silica between $0.5 \%$ and $5 \%$ does not have a discernible effect on the functional performance of the coatings. Averaged over the wear cycles, the contact angle is lowest at $10 \%$ and highest at $2 \%$ and $3 \%$ for coatings with added colloidal silica. However, the differences in the contact angles between the coatings at the highest penetration depths recorded are in a practical sense insignificant. 


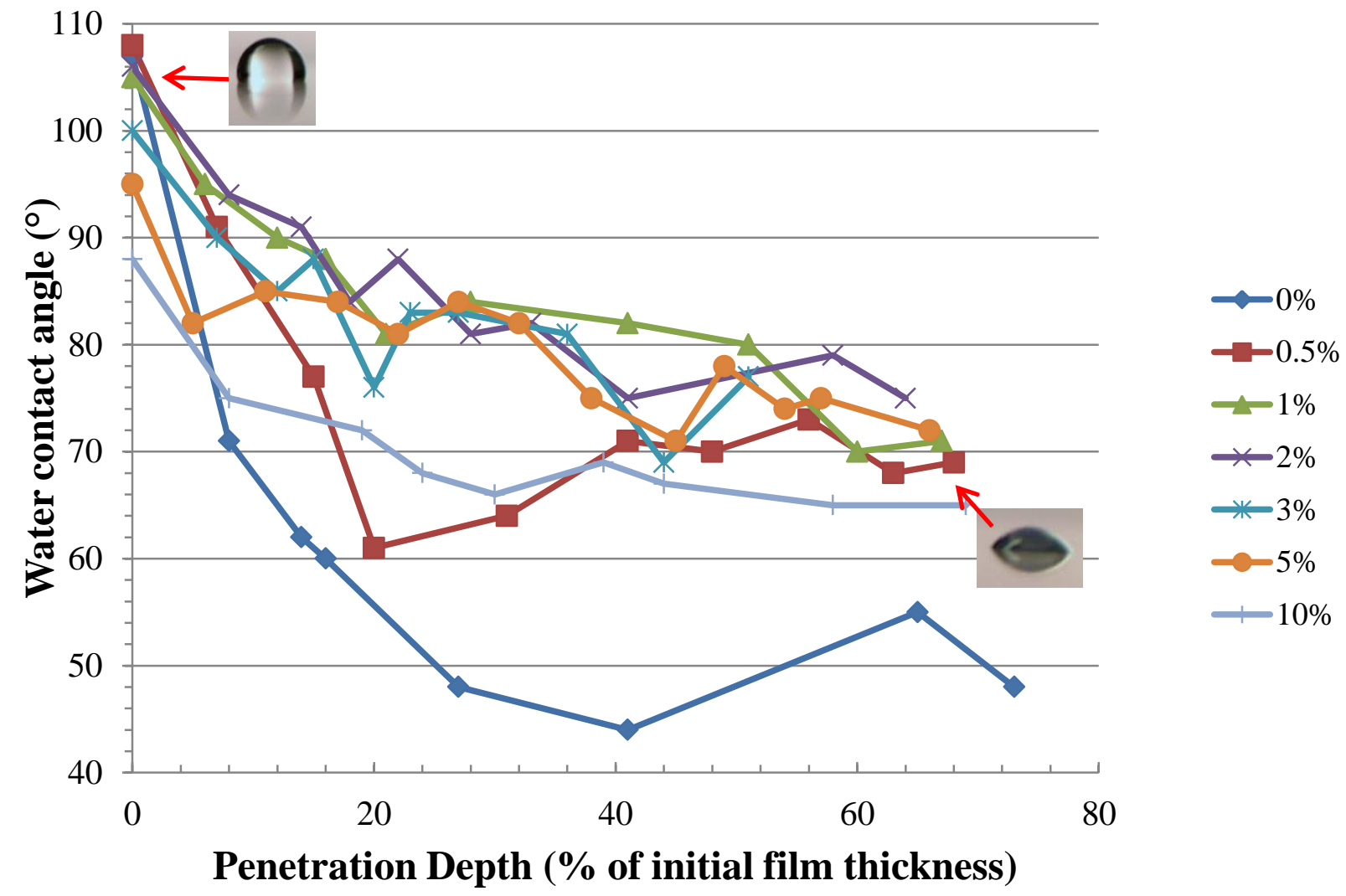

Figure 27: Contact angle of non-templated coatings vs. the penetration depth into the coating as a percent of the initial film thickness 


\subsection{Mechanical and Tribological Analysis}

\subsubsection{Abrasive Wear Tester}

To determine the wear resistance of the coatings, each was tested under the abrasive wear tester as described previously. The wear rates of the non-templated coatings were calculated as the average mass loss per cycle. Four samples of each coating formulation were tested for statistical analysis. The thicknesses of the coatings used were between 600 and $700 \mathrm{~nm}$, dipped with a withdrawal speed of $200 \mathrm{~mm} / \mathrm{min}$.

As stated previously, the roughness $\mathrm{R}_{\mathrm{a}}$ of each pristine coating was below $5 \mathrm{~nm}$ up to a loading of $3 \%$ added colloidal silica to the non-templated coatings. At this point, $\mathrm{R}_{\mathrm{a}}$ values increased to between $10-15 \mathrm{~nm}$, with no discernible difference in the $5 \%$ and $10 \%$ added silica coatings. After any number of abrasive wear cycles, the roughness values measured over a $1 \mathrm{~mm}$ scan on the stylus profilometer further increased to between 10 and $20 \mathrm{~nm}$ for coatings with $2 \%$ or less added colloidal silica. For coatings with $3 \%$ or more added colloidal silica, this $\mathrm{R}_{\mathrm{a}}$ increased further to between 20 and $25 \mathrm{~nm}$. This distinct separation in measured roughness values is likely attributed to a larger particle size on the surface due to agglomeration of the silica nanoparticles in the sol and surface segregation. These profilometry results agree with the findings from atomic force microscopy.

Figure 28 shows the wear rates calculated for each non-templated coating formulation. The graph shows a distinct decline in wear rate as the concentration of added colloidal silica increases to $1 \%$ and $2 \%$. However, at concentrations higher than $2 \%$, the wear rate is higher than at $1 \%$ and $2 \%$ but still lower than $0 \%$ and $0.5 \%$. Thus there is an optimum concentration of $1 \%$ and $2 \%$ added colloidal silica for wear rate characteristics. This increase in wear at higher 
concentrations can be attributed to the increased roughness and porosity leading to coating delamination after even a small number of wear cycles as seen in previous work by Kessman et al. [7].

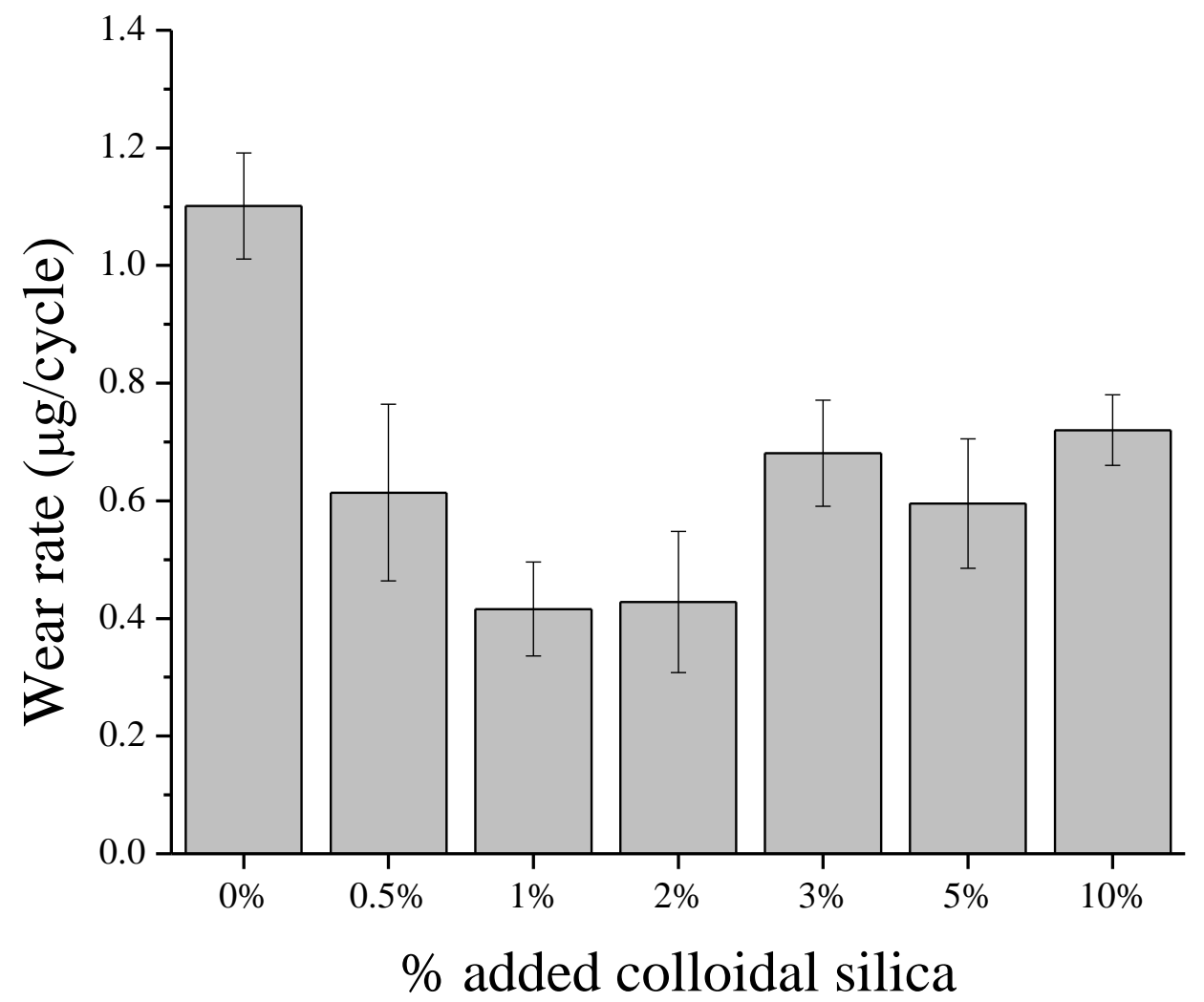

Figure 28: Wear rate calculated from mass loss of non-templated coatings with added colloidal silica

The wear rates for the templated coatings with added colloidal silica were calculated using the Archard equation (Equation 2, Section 3.2.5) and are shown in Figure 29. This wear rate, as previously stated, is calculated as the volume of the coating lost (as measured from thickness loss) normalized by the sliding distance and applied normal load. 
The average roughness, $R_{a}$, values of each pristine coating were measured similarly to the non-templated coatings. The $0 \%, 0.5 \%$, and $1 \%$ added colloidal silica coatings generally had roughness values between 10 and $15 \mathrm{~nm}$. This is a significant increase (from $5 \mathrm{~nm}$ ) from the nontemplated coatings. $\mathrm{R}_{\mathrm{a}}$ values of concentrations above $1 \%$ further increased to between 20 and 25 $\mathrm{nm}$. This is again likely due to an even greater increase in particle size on the surface due to the addition of surfactant. After abrasive wear, however, these roughness values did not increase beyond $25 \mathrm{~nm}$ for any coating. Again, these profilometry results agree with the findings from atomic force microscopy.

Similarly to the non-templated coatings, there is a slight decrease in the wear rate as the concentration of colloidal silica is increased. However, initially at $0.5 \%$ added silica, there is a slight increase in wear rate attributed to an increase in porosity and roughness from the colloidal silica. Thus the addition of colloidal silica can be detrimental to the abrasive wear resistance of a template coating if the additional porosity is not overcome mechanically. The lowest wear rates were consistently at concentrations of $2 \%$ and $3 \%$ added silica, and the wear rates at higher concentrations were generally indiscernible from the $1 \%$ and below. Thus the optimum concentration of added colloidal silica was found to be between $2 \%$ and $3 \%$. 


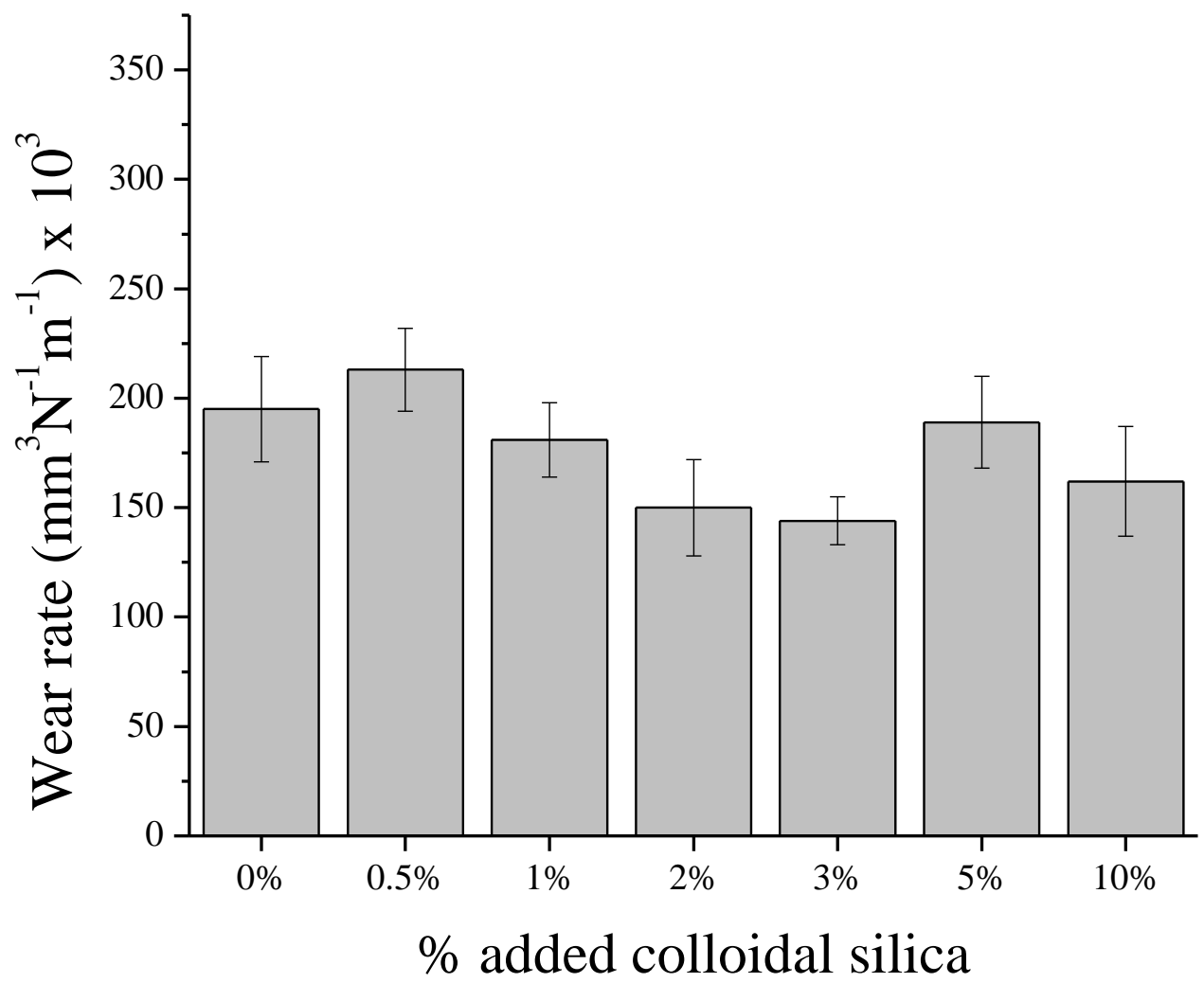

Figure 29: Wear rate of templated coatings with added colloidal silica using the Archard equation 


\subsubsection{Linear Reciprocating Tribometer}

The CSM Instruments Micro-Tribometer was used to test non-templated coatings with $0 \%$ and $2 \%$ added colloidal silica, as these have been shown to be the two polarizing samples through other characterization. Sample results from the CSM Instruments Micro-Tribometer are shown in Figure 30. This figure shows the coefficient of friction plotted against the wear cycles and is representative of the measured coefficient of friction for both coatings. The absolute value of the maximum and minimum peaks of this graph correspond to the calculated coefficient of friction as the sample is in linear motion relative to the static steel ball contact. Figure 30 shows that this absolute value of the coefficient of friction increases from an initial value of approximately 0.2 on the first cycle to a final maximum of 0.4 after about 7 cycles. This was the typical result with the final maxima ranging between 0.4 and 0.5 for each run. These values are consistent with other similar experiments done by López et al. [29] on a pin-on-disk tribometer with nearly identical conditions. 


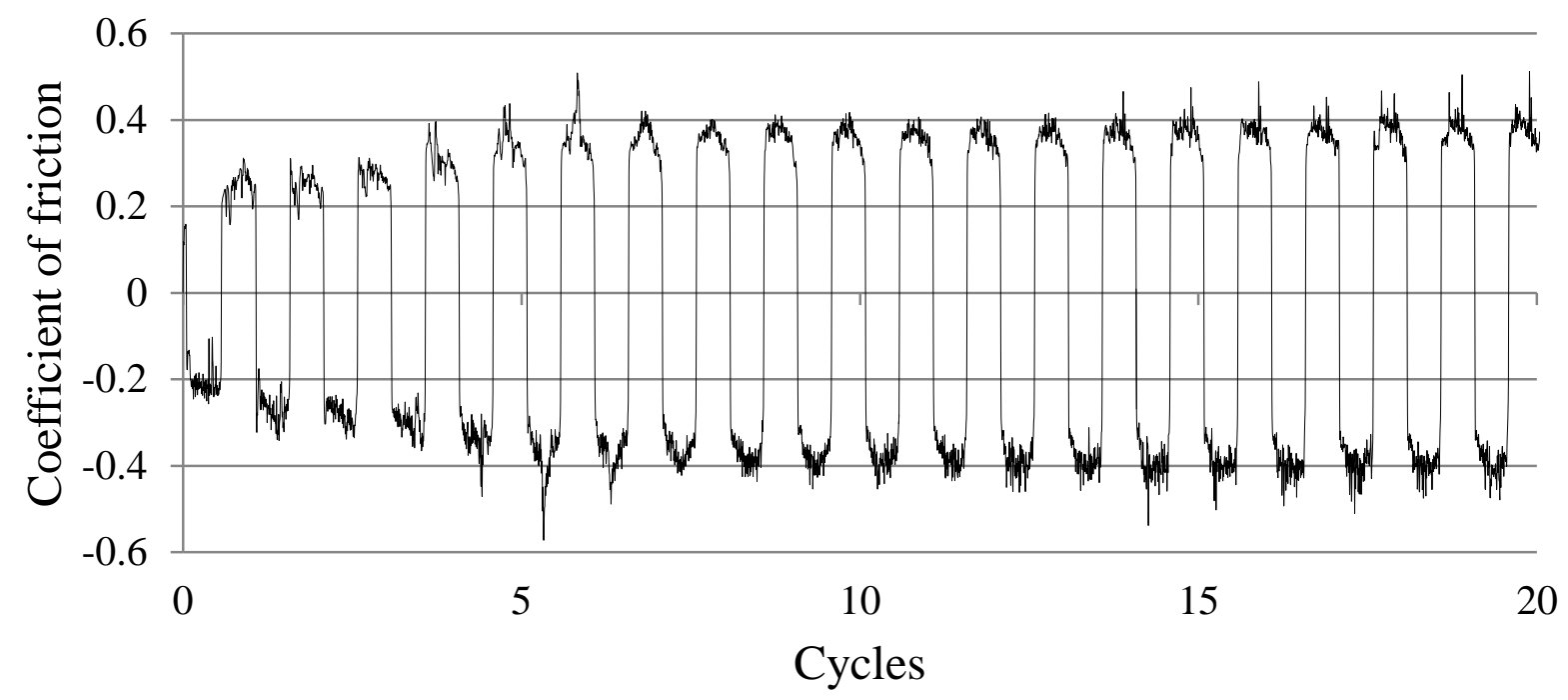

Figure 30: Coefficient of friction output vs. wear cycles of non-templated coating with $2 \%$ added colloidal silica using CSM Instruments Micro-Tribometer

Optical microscopy was also performed to analyze the wear tracks and to determine the wear mechanisms. Figure 31 shows these optical micrographs for the $0 \%$ and $2 \%$ added colloidal silica coatings. For both samples, there were clearly apparent abrasion lines along the wear track parallel to the sliding direction. Additionally, both coatings have detached material and debris typical of abrasive and surface fatigues wear mechanisms. Also, the coatings were still left mostly intact as shown by the profilometry tracks across the wear tracks.

However, the $0 \%$ added colloidal silica coating (Figure 31a) did not show nearly as much evidence of fragmentation and spallation as the $2 \%$ added colloidal silica (Figure $31 \mathrm{~b}$ ). This can likely be attributed to the larger thickness of the $2 \%$ added silica coating compared to the $0 \%$ added silica coating. This led to more of an apparent adhesive failure rather than abrasive wear failure of the $0 \%$. 


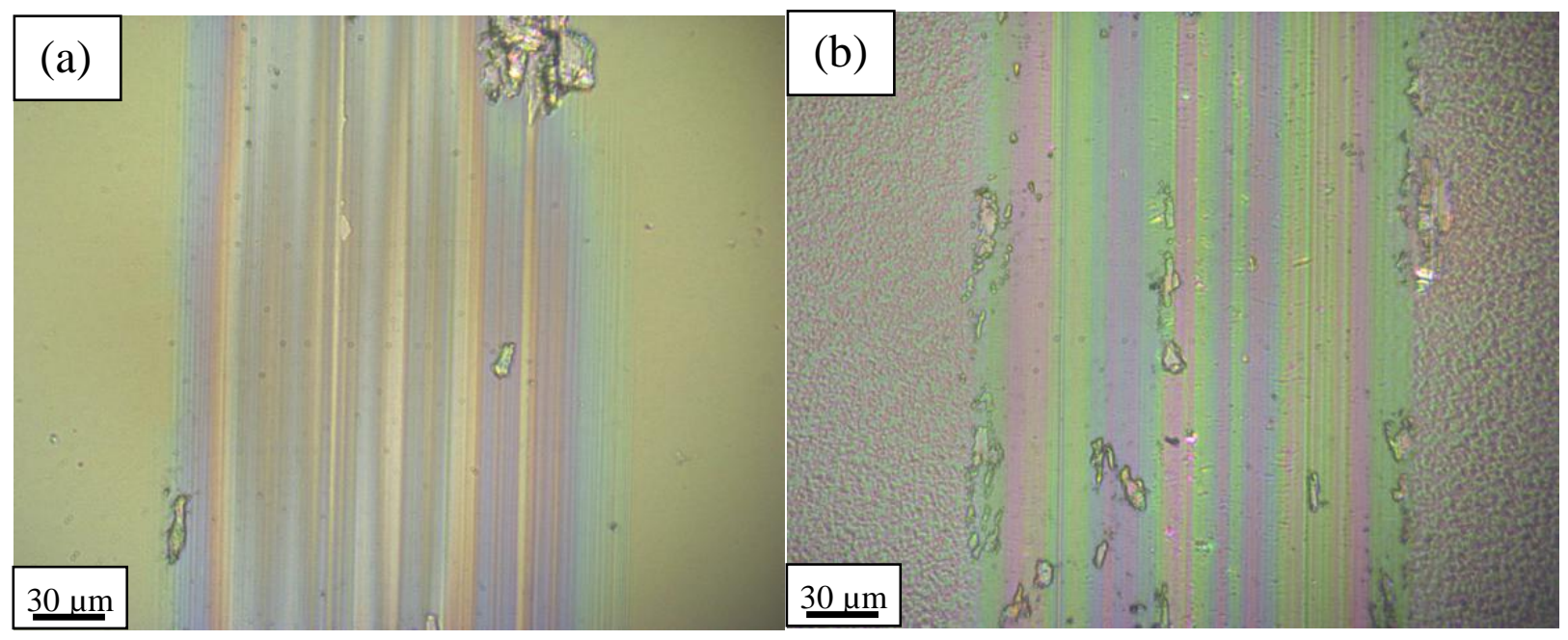

Figure 31: Optical micrographs of wear tracks of non-templated coatings with (a) $0 \%$ and (b) $2 \%$ added colloidal silica 


\subsubsection{Hardness and Elastic Modulus}

Instrumented nanoindentation using CSM Instruments’ NHT Nanoindentation Tester and UNHT Ultra Nanoindentation Tester systems were used to analyze the hardness and elastic moduli of non-templated and templated coatings with added colloidal silica. Since the NHT system was readily available at WVU, it was used for the majority of the characterization. Figure 32 shows a representative normal load - penetration depth curve from a UNHT standard indentation protocol on a non-templated coating with $2 \%$ added colloidal silica. This protocol was used with a Berkovich indenter tip with both the UNHT and NHT and with a $10 \mu \mathrm{m}$ radius sphero-conical tip with the NHT.

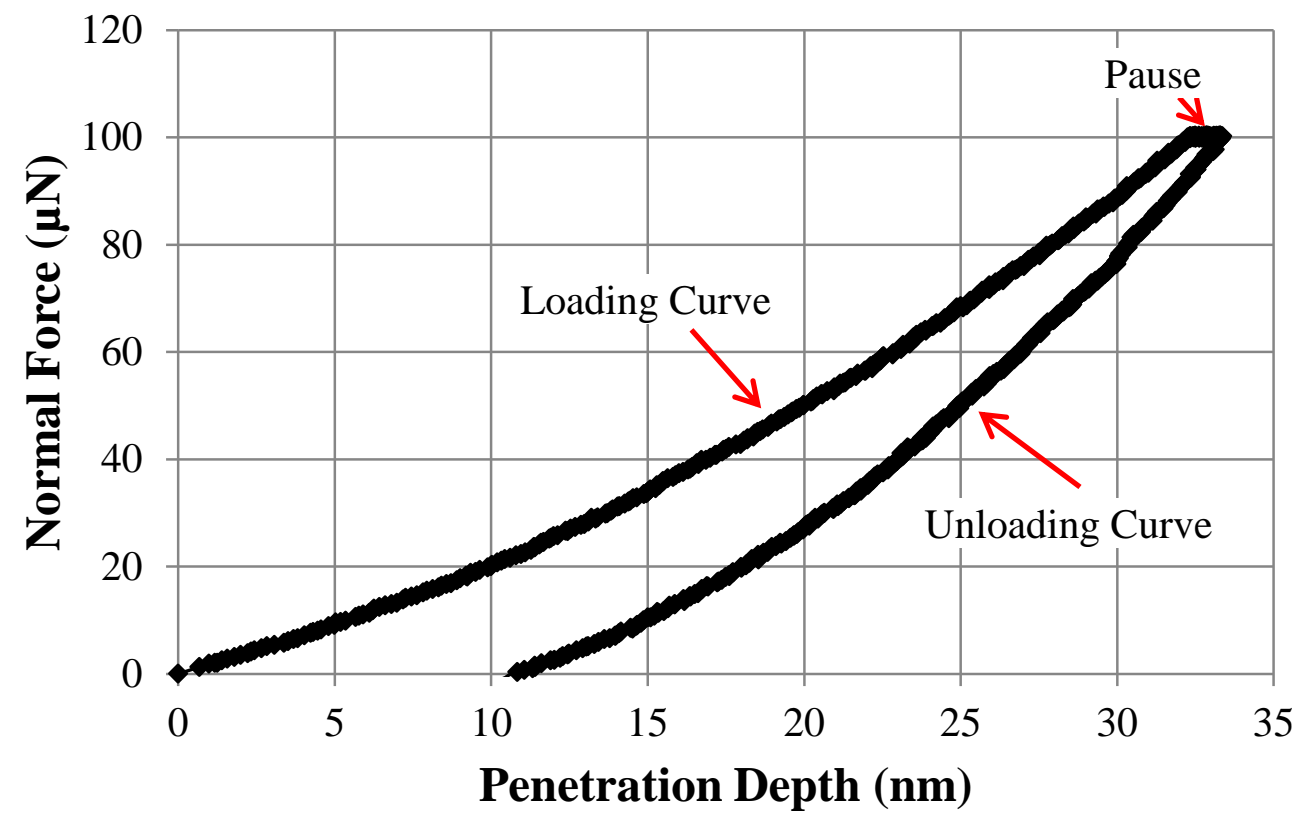

Figure 32: Representative normal load-penetration depth curve of a standard indentation protocol performed on particle-reinforced coatings with UNHT 
The Berkovich indenter tip was used first to measure the hardness and elastic moduli of the non-templated coatings. The results of these are shown in Figure 33. The highest hardness values measured were $1 \%, 2 \%$, and $3 \%$ added colloidal silica. The hardness values doubled from $0 \%$ added silica to $2 \%$. After this point, however, the hardness values decreased slightly, similarly to the abrasive wear resistances found previously. For comparison purposes, the hardness values were plotted on the same graph as the mass loss wear rates in Figure 34. As expected, this graph shows an inverse relationship between the indentation hardness and the wear rate. Thus with improved hardness, the abrasive wear resistance of the coatings increases. Similarly to the abrasive wear testing results, there is an apparent detrimental effect on the strength of the coating above a certain concentration of added colloidal silica. This concentration for the non-templated coatings is consistently around $2 \%$. 


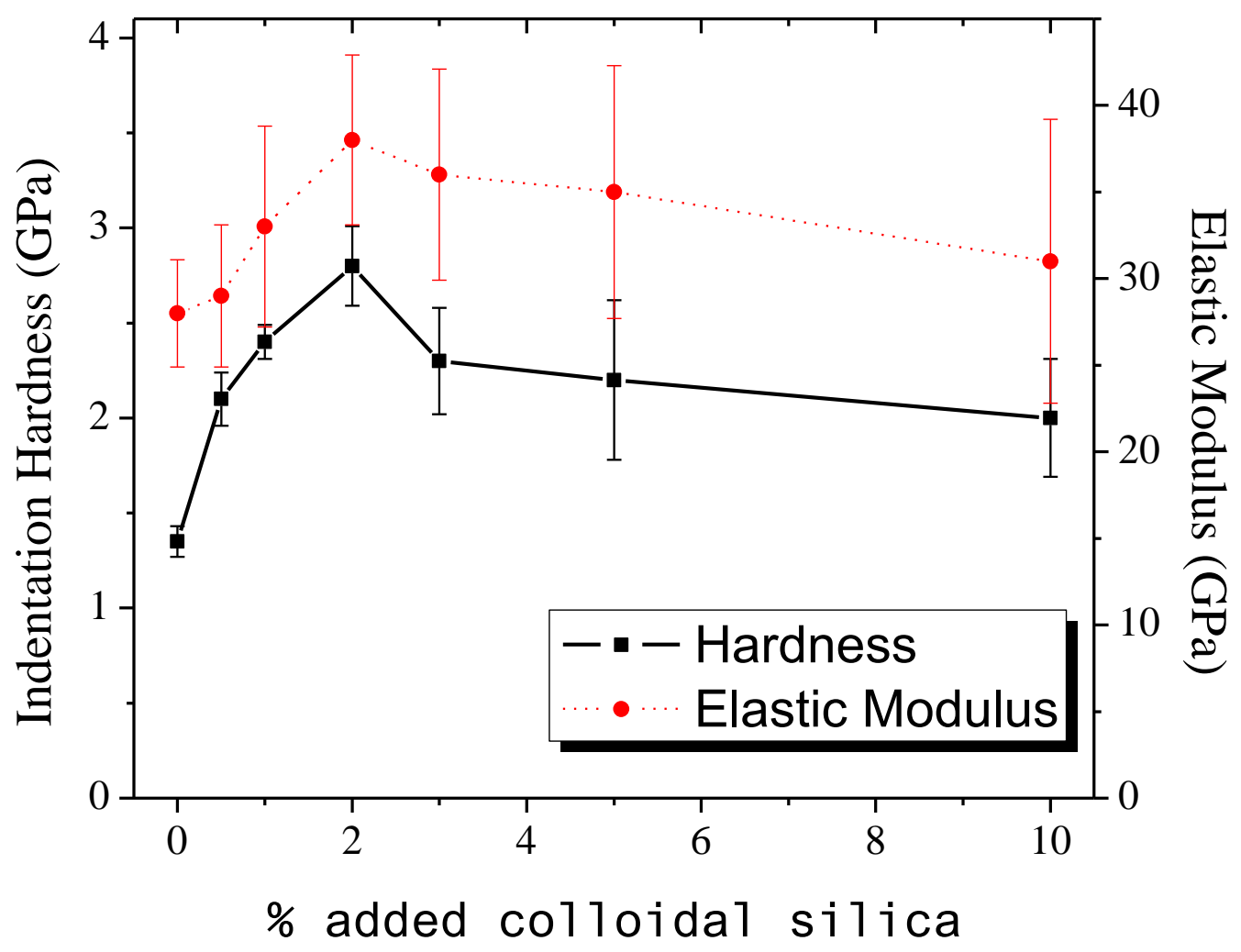

Figure 33: Indentation hardness and elastic moduli of non-templated coatings

In addition to the hardness, the elastic modulus is shown in Figure 33. As expected, these results follow the same trend as the hardness but with a lesser increase in magnitude from the coatings with $0 \%$ to coatings with $2 \%$ added colloidal silica. The elastic modulus is similarly affected by the porosity induced by added silica, so it stands that there would be an optimum concentration of colloidal silica as with hardness.

The higher roughness at higher concentrations of added silica affects the distribution of moduli depending on the intender tip impact with a single asperity on the coating. For this reason, it is preferred to use a sharp tip with well-defined geometry (like a Berkovich tip) and to penetrate a surface well beyond the average surface roughness (generally 10-20 times). However, 
the high roughness of the higher added silica concentrated coatings present a challenge in achieving a low standard deviation with these coatings and reliable results.

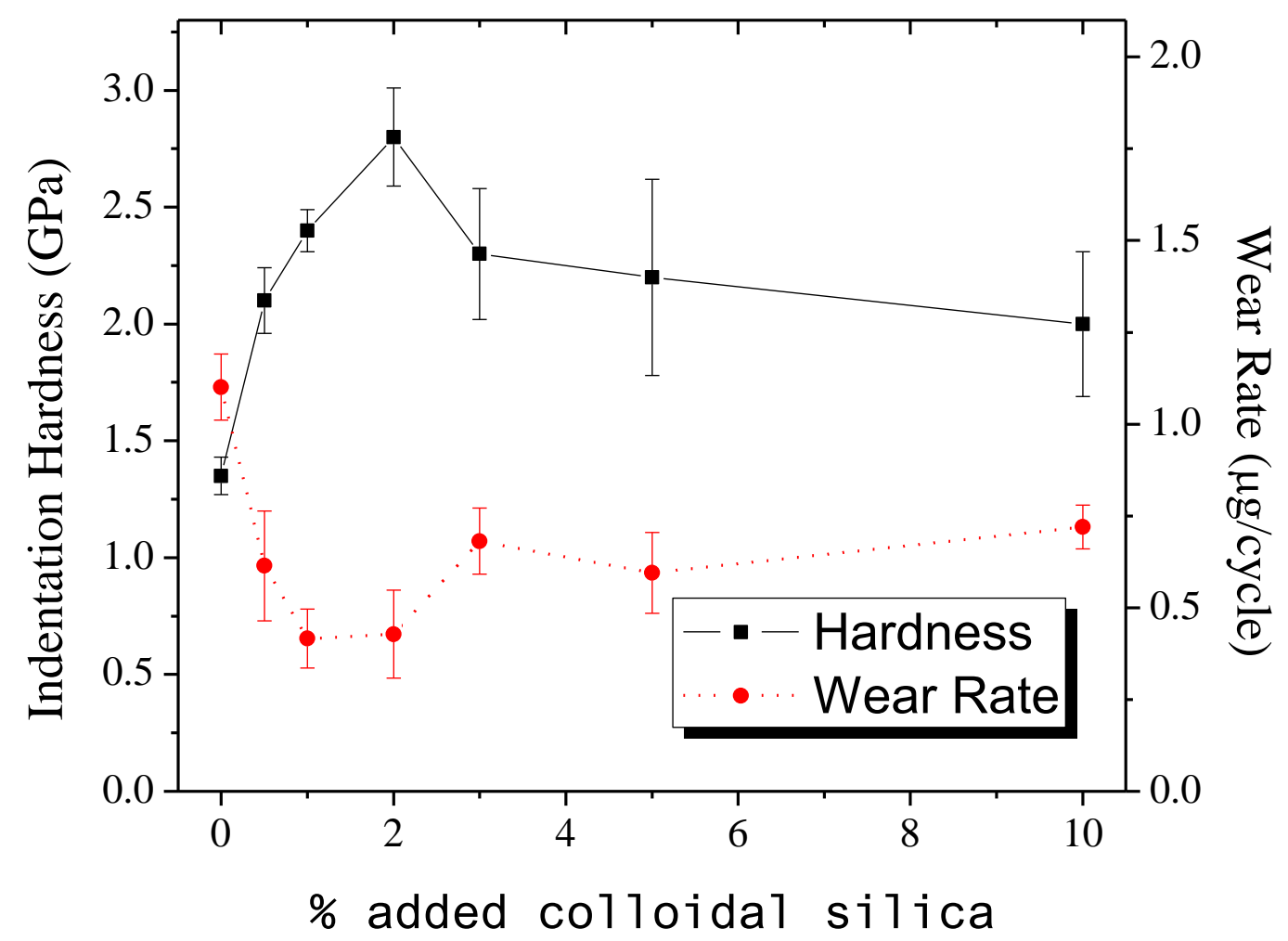

Figure 34: Indentation hardness and wear rates of non-templated coatings

The templated coatings were tested with the NHT and the results are shown in Figure 35 with the Archard equation derived wear rates. The elastic moduli for the templated coatings could not be reliably measured due to their high average roughness values and reasons stated previously. The trend in hardness values with the template coating is slightly shifted to 2-3\% being the optimum concentrations of added colloidal silica, compared to 1-2\% for non-templated coatings. The hardness values at $2-3 \%$ are double that of those of $0 \%$ added colloidal silica. Additionally, the wear rates are lowest at these higher hardness values as expected. Similarly to 
the non-templated coating at higher concentrations than $3 \%$, the hardness values are slightly lower due to the surface roughness and porosity.

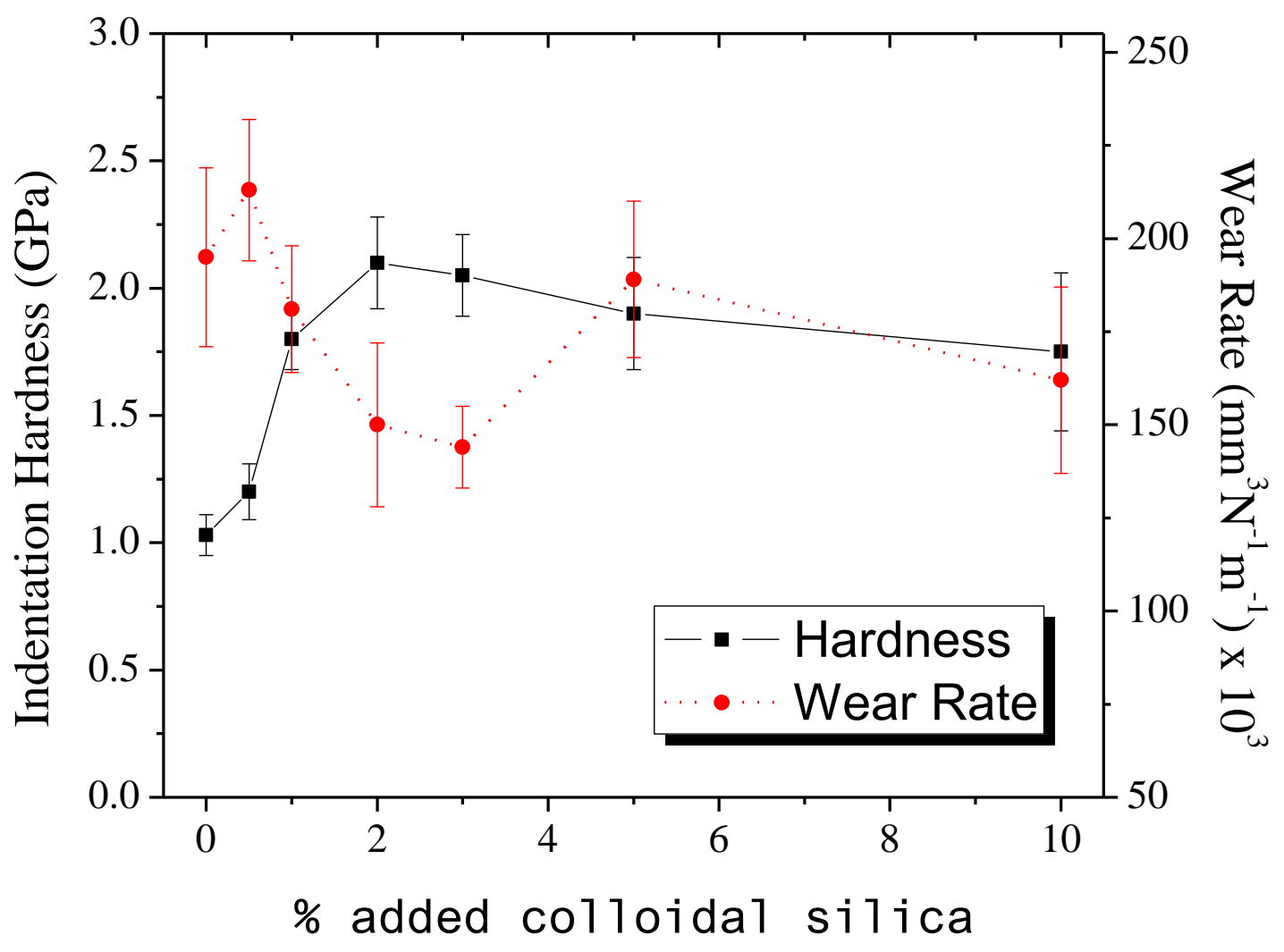

Figure 35: Indentation hardness and wear rates of templated coatings

To investigate the extent of the substrate effect on the hardness and modulus measurements of the coatings, a constant multicycle (CMC) indentation protocol was used. A characteristic normal load - penetration depth curve is shown in Figure 36 for this CMC protocol on a non-templated $2 \%$ added colloidal silica coating. The thickness of this coating was $650 \mathrm{~nm}$, and as shown the maximum penetration depth at the final maximum load was $265 \mathrm{~nm}$, or about $40 \%$ of the thickness of the coating. At this point, the hardness value measured should be extremely affected by the soda-lime glass substrate. The hardness values calculated from this 
protocol for the $2 \%$ added silica coating for these 7 separate indents in the CMC protocol are shown in Figure 37. Compared to the measured hardness of the plain soda-lime glass slide of about 7.6 GPa, the hardness at a penetration depth of $10 \%$ of the thickness of the coating is 3.1 GPa. On the final load up to a penetration depth of $40 \%$ of the coating thickness, this hardness value increases to $4.8 \mathrm{GPa}$. This is more than a $50 \%$ increase in magnitude, and thus it is absolutely imperative to abide by the guideline of penetrating below $10 \%$ of the total thickness for these "soft-on-hard" coating-substrate systems.

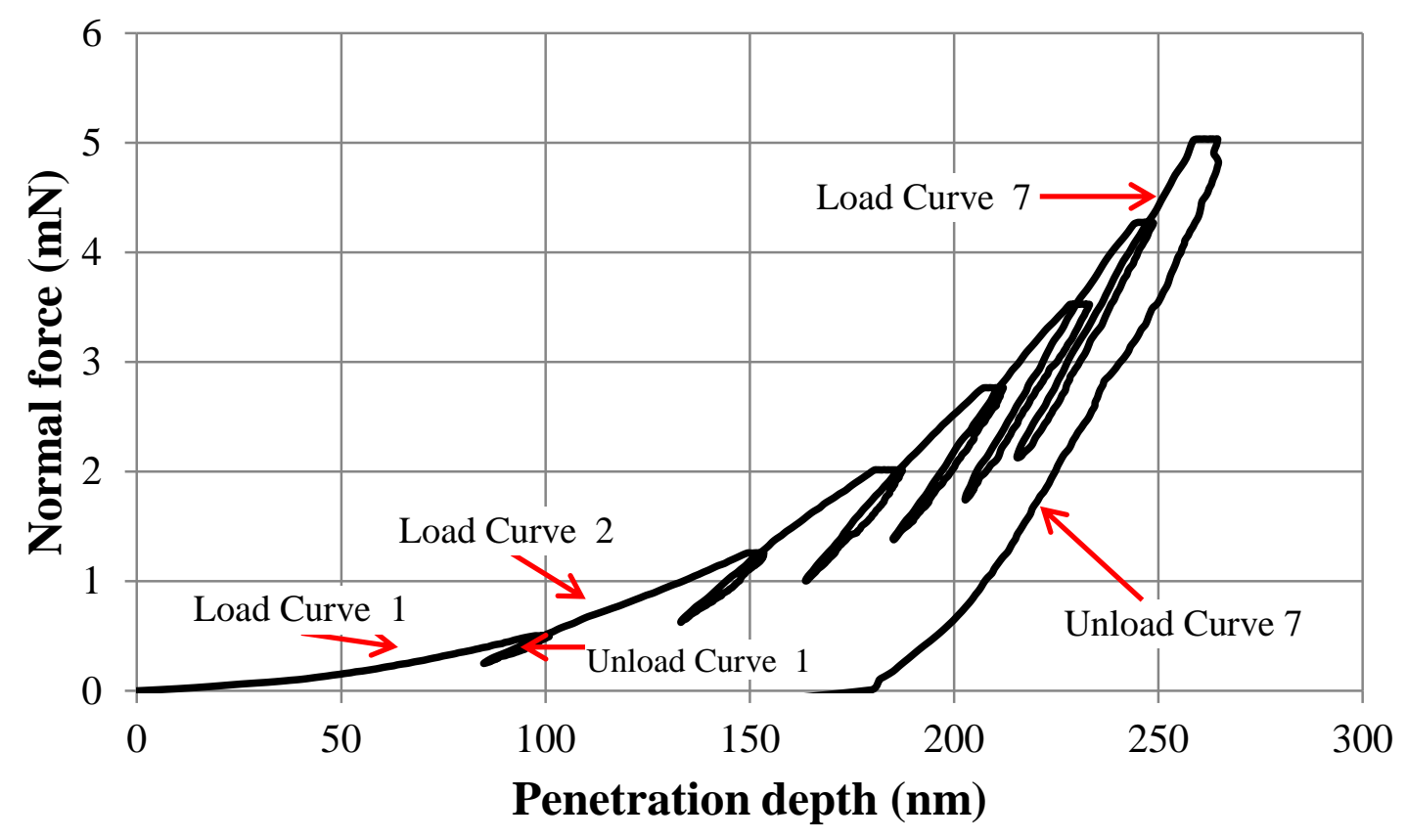

Figure 36: Indentation curve for a continuous multicycle indentation protocol on a nontemplated coating with $2 \%$ added colloidal silica 


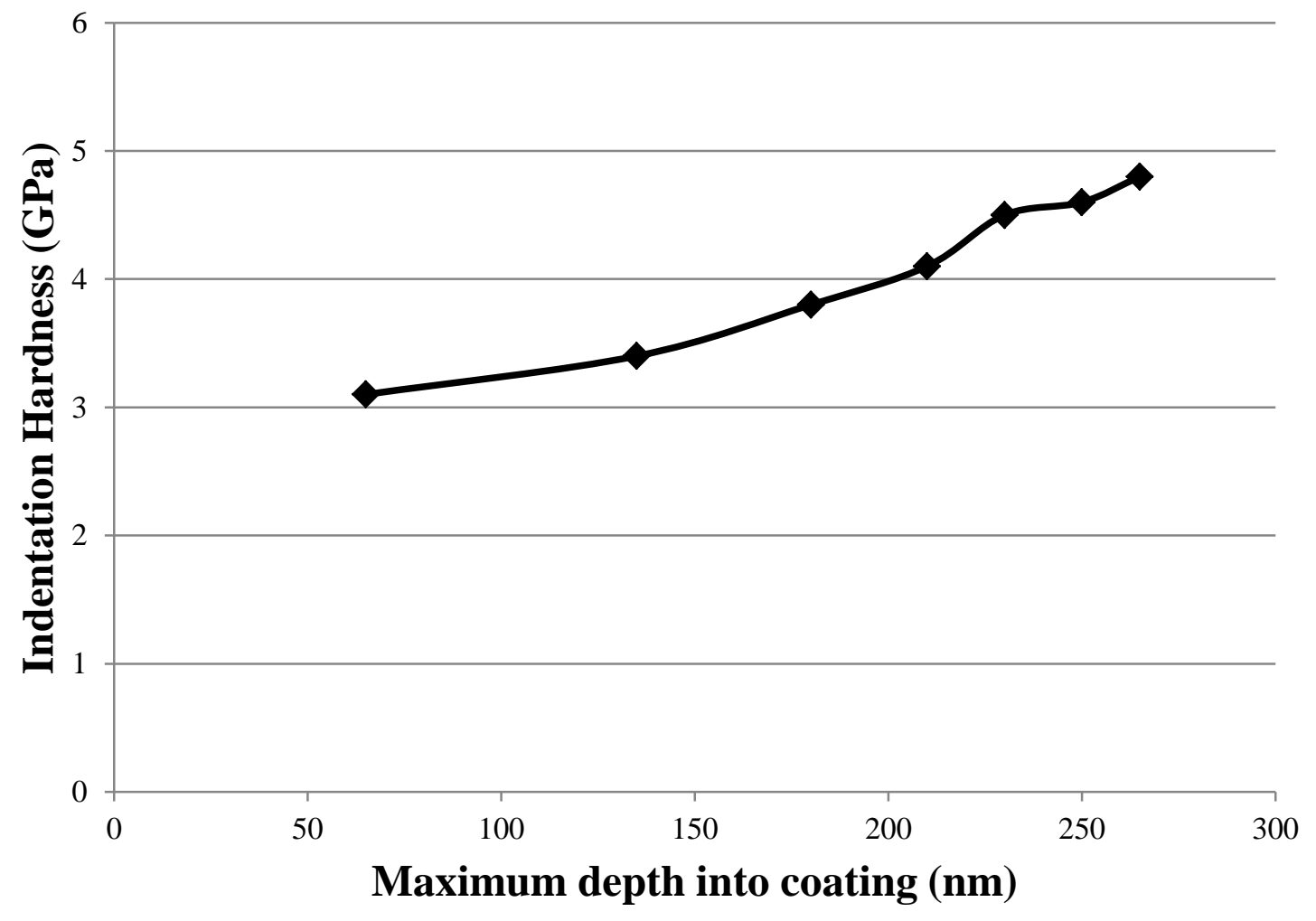

Figure 37: Hardness of a non-templated 2\% added colloidal silica coating as a function of penetration depth into the coating 


\subsubsection{Coating Adhesion/Cohesion}

The nanoscratch testing performed on the non-templated $0 \%, 1 \%$, and $2 \%$ added colloidal silica coatings gave insight into the adhesion strength and adhesive failure mechanisms of the coating. Figure 38 shows the panoramic optical micrographs of the entire length of the $500 \mu \mathrm{m}$ scratches. The red arrows on the panoramas signify the critical failure loads, labeled as $\mathrm{L}_{\mathrm{C} 1}$ and $\mathrm{L}_{\mathrm{C} 2}$. The average normal load values of $\mathrm{L}_{\mathrm{C} 1}$ and $\mathrm{L}_{\mathrm{C} 2}$ for the $0 \%$ and $1 \%$ were practically indistinguishable at around $19 \pm 1 \mathrm{mN}$ and $36 \pm 3 \mathrm{mN}$, respectively. The $\mathrm{L}_{\mathrm{C} 1}$ failure mode of these two coatings can be described as a cohesive failure with plastic deformation as shown in (a) of both Figure 39 and Figure 40. There is no noticeable cracking of the coating prior to these loads

most likely due to their low thickness. The $\mathrm{L}_{\mathrm{C} 2}$ failure mode of these coatings is best described as a cohesive complete delamination as shown magnified in (b) of Figure 39 and Figure 40. 


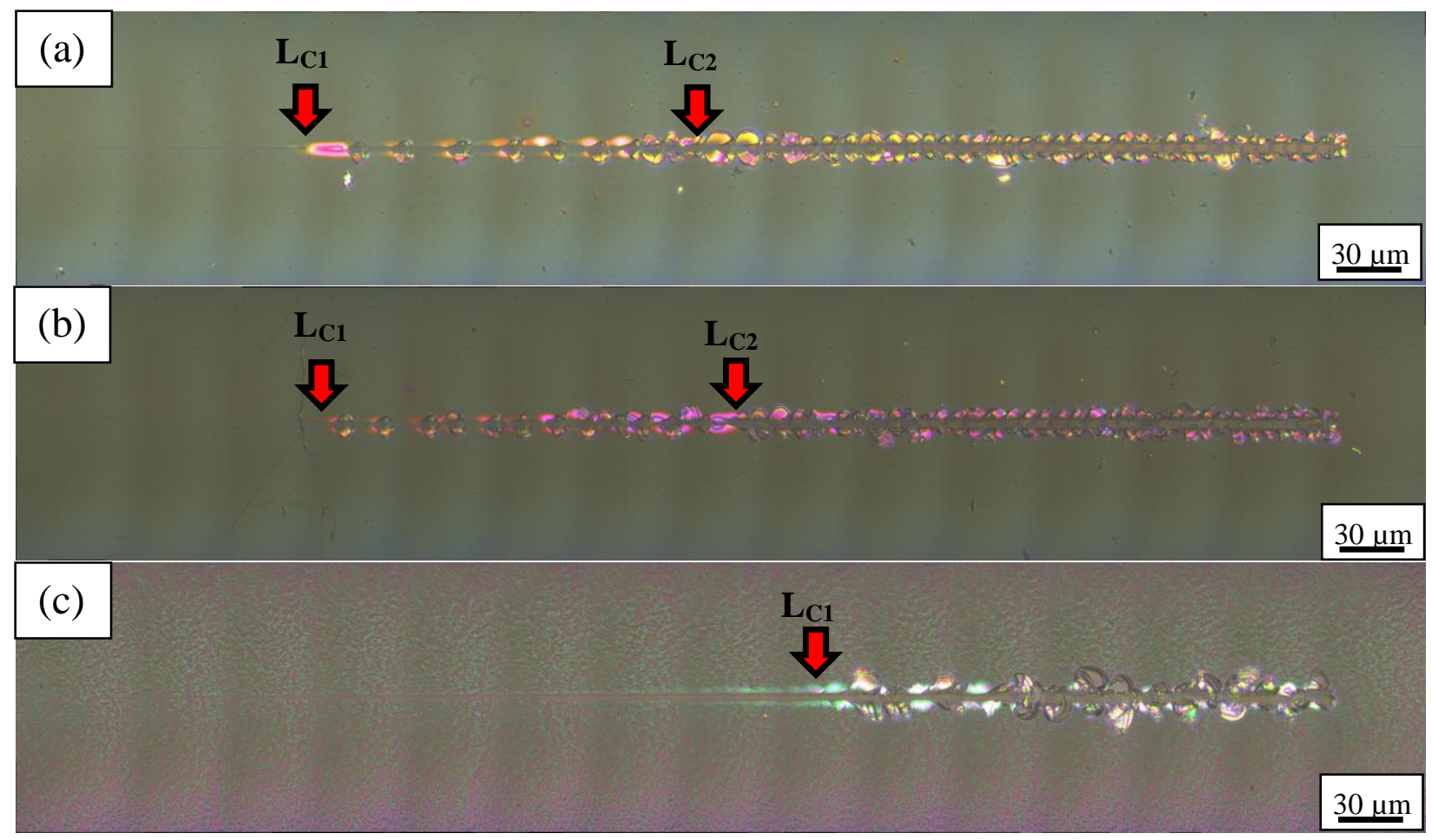

Figure 38: Panoramic optical micrographs of nanoscratch test on (a) $0 \%$, (b) $1 \%$, (c) $2 \%$ added colloidal silica. Arrows signify critical failure loads.
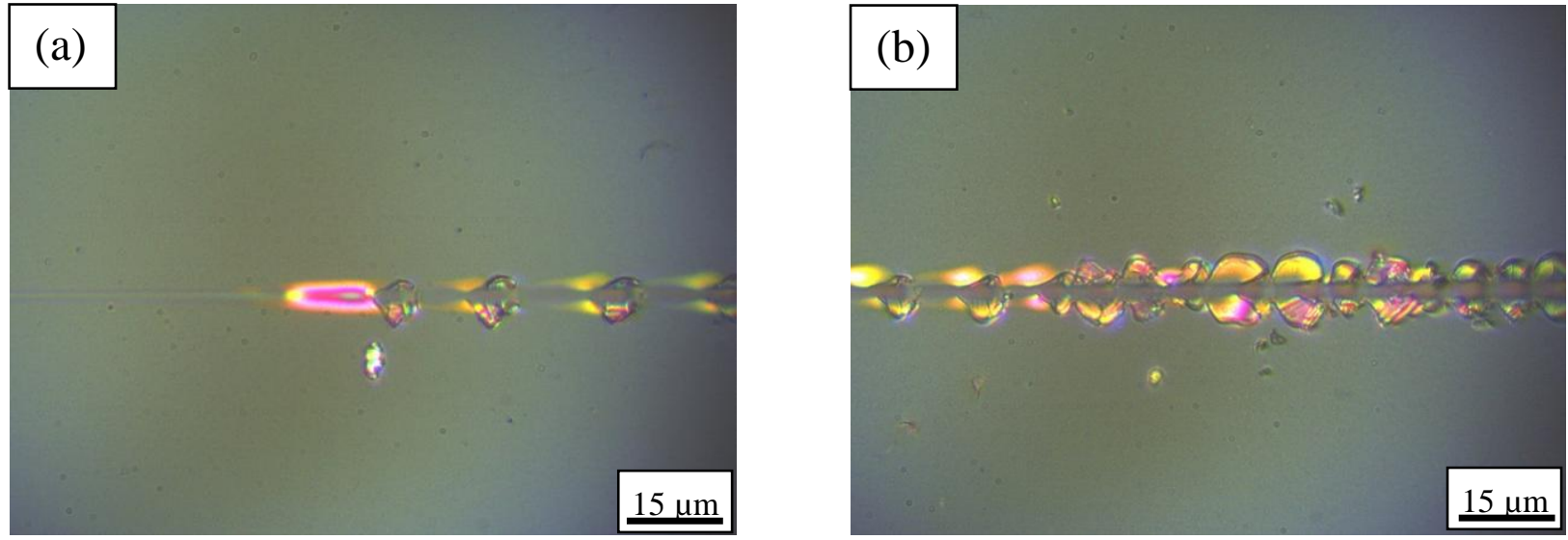

Figure 39: Optical micrograph of (a) $L_{C 1}$ and (b) $L_{C 2}$ of a non-templated coating without added silica. 

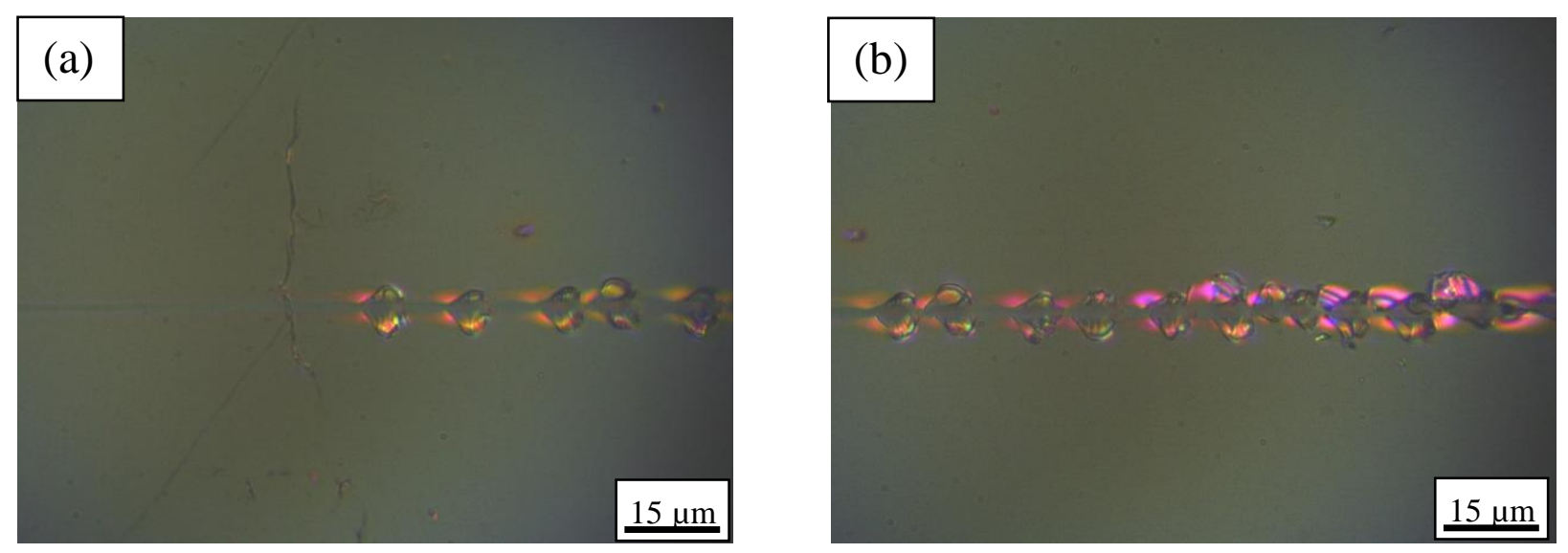

Figure 40: Optical micrograph of (a) $L_{C 1}$ and (b) $L_{C 2}$ of a non-templated coating with $1 \%$ added colloidal silica.

Figure 38(c) differs greatly from (a) and (b) in that there is only one observable critical failure load. This $\mathrm{L}_{\mathrm{C} 1}$ is best characterized as an adhesive failure of spalling and delamination, as shown magnified in Figure 41. The average normal load for this to occur was $44 \pm 3.5 \mathrm{mN}$. This higher normal load value can likely be attributed to a harder and more wear-resistant surface than the coatings with less added colloidal silica. A potential cause for the difference in apparent failure mechanisms could be due to additional porosity and thickness from the added colloidal silica. 


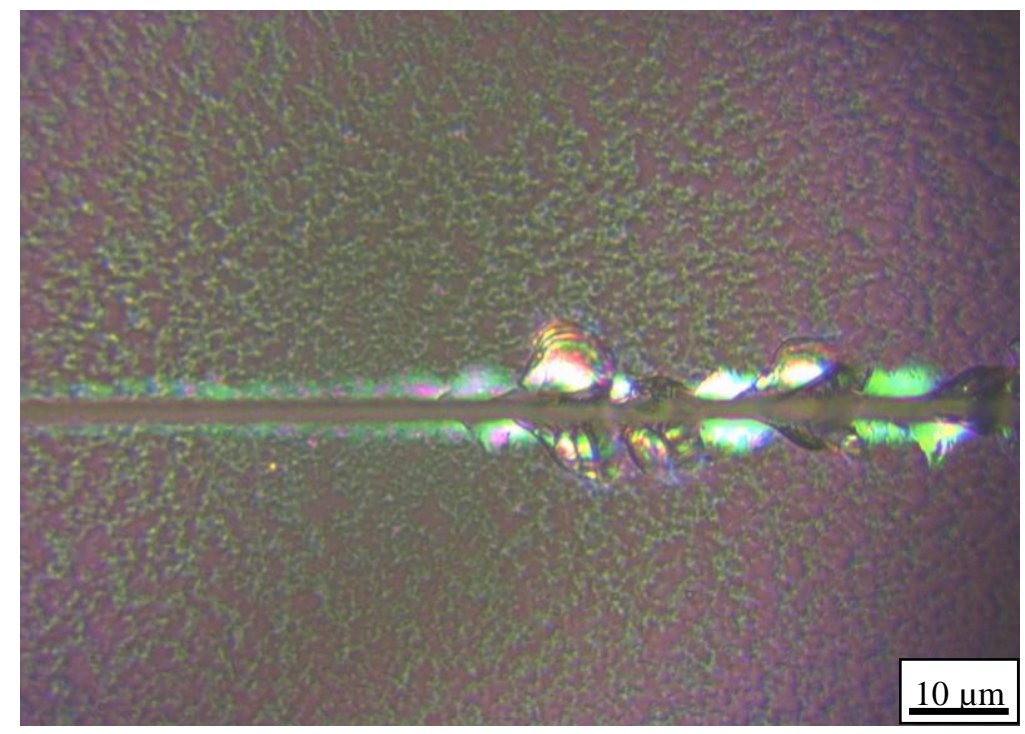

Figure 41: Optical micrograph of $\mathrm{L}_{\mathrm{C1}}$ of a non-templated coating with $2 \%$ colloidal silica. 


\section{$5 \quad$ Summary and Conclusions}

The overall goal of this research study was to synthesize widely applicable and adaptable sol-gel coatings that were hydrophobic, thin, wear-resistant, thermally stable, transparent, and easily deposited. While most of these objectives are interrelated and require compromises to achieve simultaneously, the sol-gel method is exceptionally useful since it allows for tailoring and optimization depending on the final application and required performance.

The primary objective of achieving a wear-resistant hydrophobic coating through colloidal silica nanoparticle matrix reinforcement was achieved by examining the effect of varying concentrations of colloidal silica. Furthermore, the coatings were synthesized with and without a templating surfactant to elucidate the effect of added porosity on the mechanical and tribological properties of the coatings.

By building upon and altering previous formulations in literature (primarily by Cairns and Kessman et al. $[5,7,10,11])$, the coatings were synthesized, dip-coated, and cured at $200^{\circ} \mathrm{C}$ on soda-lime glass slides with final thicknesses between 500 and $700 \mathrm{~nm}$. The coatings were then characterized using several different instruments and methods. These included optical transmission and microscopy, atomic force microscopy, stylus profilometry, contact angle goniometry, an in-lab built mild abrasive wear tester, an industrial linear reciprocating microtribometer, two nanoindentation systems, and a nanoscale diamond-stylus scratch tester. These different instruments were used to characterize the overall optical, structural, mechanical, functional, and tribological properties of the coatings.

The optical analyses of the coatings showed that they were all relatively optically transmissive. All coatings with concentrations below 5\% added colloidal silica had average 
transmissions across the visible spectrum equal to or better than the bare glass slide. The structural analysis using atomic force microscopy showed that the average roughness of the coatings increased significantly at $3 \%$ added colloidal silica to about $15 \mathrm{~nm}$ for the nontemplated coatings. Also for templated coatings, this roughness was equal at $2 \%$ added colloidal silica. Overall, the roughness of the coatings increased as expected with increased concentration of colloidal silica. It is concluded that the nanoparticles (average diameter of $20 \mathrm{~nm}$ in suspension) aggregated and surface segregated at these higher concentrations, and this is further confirmed through abrasive wear and indentation testing.

The functionality of the coatings was characterized and quantified using contact angle goniometry. The wetting of the sample surface by water was measured before and after abrasive wear by the in-lab built abrasive wear tester. The highest functional sustainability averaged throughout the wear of the coatings was achieved at $2 \%$ and $3 \%$ added colloidal silica, but there was no discernible difference between any of coatings with concentrations between $0.5 \%$ and $5 \%$ in a practical sense. It can be concluded that the concentration of fluorosilanes was sufficiently high enough to be encapsulated within the induced porosity from the colloidal silica. The water contact angles for these coatings between $0.5 \%$ and $5 \%$ were consistently between $70^{\circ}$ and $90^{\circ}$ after any number of wear cycles. Of these, all except $5 \%\left(95^{\circ}\right)$ had water contact angles above $100^{\circ}$ on pristine surfaces before wear.

The mechanical and tribological analyses of the coatings mainly involved quantifying the wear rates through an in-lab built mild abrasive wear apparatus and comparing these to measured indentation hardness. These wear rates were calculated in terms of mass loss and volumetric loss. Overall, the most favorable results for non-templated coatings were achieved with 1-2\% added colloidal silica, with an increase in wear rate above these concentrations. With the surfactant 
templating, the wear rate was optimum at the $2-3 \%$ concentration. As expected, the indentation hardness and elastic moduli were generally the highest at the same concentrations that had the lowest wear rates. Similarly to other characterization methods, surface segregation and particle aggregation affected results of the higher concentrations of added colloidal silica.

Complementary analyses of the coating adhesion, friction, and wear mechanisms were performed to confirm these other mechanical and tribological studies. These studies involved a nanoscratch testing system and a linear reciprocating micro-tribometer. Overall, the addition of colloidal silica to the sol-gel formulation increased the critical load of adhesive failure significantly. There were multiple wear mechanisms involved in the abrasive wear of these coatings, all apparent factors of the induced porosity and roughness due to added colloidal silica and surfactant templating.

While the ability to tailor the properties of these durable sol-gel derived silica coatings was achieved, there are still many possibilities for further work on this subject. The overall goal of deconvoluting the effects of the colloidal silica and surfactant can be further explored through porosity and sol particle size characterization as well as through a more refined tribological characterization method. The effect of sol aging on the microstructure and properties of the deposited sol-gel matrix can be investigated further for these multiphase coatings. Also, alternatives to presently used fluorosilanes or surfactants could be used, including a combination of smaller molecular weight surfactants and swelling agents or different sized colloidal silica nanoparticles to reduce the induced porosity.

Other possibilities for further work include expansion into other functionalities in addition to or in replacement of hydrophobicity. As previously noted, these applications could 
include antifouling, anti-corrosive, antimicrobial, and anti-icing. Expansion into different deposition methods such as spray coating and spin coating could be explored as well. Overall, the study of incorporating mechanically robust thin functional coatings to extend the lifetime of the aforementioned applications has limitless possibilities. 


\section{References}

[1] A. Telford, M. James, L. Meagher, C. Neto, ACS Applied Materials \& Interfaces 2 (2010) 2339.

[2] K. Efimenko, J. Finlay, M. Callow, J. Callow, J. Genzer, ACS Applied Materials \& Interfaces 1 (2009) 1031.

[3] L. Cao, A. Jones, V. Sikka, J. Wu, D. Gao, Langmuir 25 (2009) 12444.

[4] G. Xue, X. Huang, J. Dong, J. Zhang, Journal of Electroanalytical Chemistry 310 (1991) 139.

[5] A. Kessman, D. Cairns, Journal of Colloid and Interface Science 360 (2011) 785.

[6] K. Tanaka, Y. Uchiyama, S. Toyooka, Wear 23 (1973) 153.

[7] A. Kessman, E. DeFusco, A. Hoover, K. Sierros, D. Cairns, Thin Solid Films 520 (2012) 3896.

[8] M. Toselli, J. Gardella, M. Messori, A. Hawkridge, F. Pilati, C. Tonelli, Polymer International 52 (2003) 1262.

[9] P. Fabbri, M. Messori, M. Montecchi, S. Nannarone, L. Pasquali, F. Pilati, C. Tonelli, M. Toselli, Polymer 47 (2006) 1055.

[10] A. Kessman, D. Cairns, Langmuir 27 (2011) 5968.

[11] A. Kessman, S. Kukureka, D. Cairns, Wear 271 (2011) 2144.

[12] P. Suegama, A. Recco, A. Tschiptschin, I. Aoki, Progress in Organic Coatings 60 (2007) 90.

[13] J.H. Hwang, B.I. Lee, V. Klep, I. Luzinov, Materials Research Bulletin 43 (2008) 2652.

[14] M. Mani, R. Pillai, Renewable and Sustainable Energy Reviews 14 (2010) 3124.

[15] H. Elminir, A. Ghitas, R. Hamid, F. El-Hussainy, M. Beheary, K. Abdel-Moneim, Energy Conversion and Management 47 (2006) 3192. 
[16] P. Hinz, H. Dislich, Journal of Non-Crystalline Solids 82 (1986) 411.

[17] C. Schelle, M. Mennig, H. Krug, G. Jonschker, H. Schmidt, Journal of Non-Crystalline Solids 218 (1997) 163.

[18] F. Bottari, P. Richter, C.-Y. Li, US Patent 6406758 (2002).

[19] F. Samson, Surface and Coatings Technology 81 (1996) 79.

[20] G. Wu, J. Wang, J. Shen, T. Yang, Q. Zhang, B. Zhou, Z. Deng, F. Bin, D. Zhou, F. Zhang, Journal of Non-Crystalline Solids 275 (2000) 169.

[21] A. Vilcnik, I. Jerman, A. Vuk, M. Kozelj, B. Orel, B. Tomsic, B. Simonic, J. Kovac, Langmuir 25 (2009) 5869.

[22] K. Johns, G. Stead, Journal of Fluorine Chemistry 104 (2000) 5.

[23] A. Cannavale, F. Fiorito, M. Manca, G. Tortorici, R. Cingolani, G. Gigli, Building and Environment 45 (2010) 1233.

[24] B. Hong, J. Han, S. Kim, Y. Cho, M. Park, T. Dolukhanyan, C. Sung, Thin Solid Films 351 (1999) 274.

[25] Z. Cui, Q. Wang, Y. Xiao, C. Su, Q. Chen, Applied Surface Science 254 (2008) 2911.

[26] T. Yoneda, T. Morimoto, Thin Solid Films 351 (1999) 279.

[27] S. Giessler, E. Just, R. Störger, Thin Solid Films 502 (2006) 252.

[28] N. Raman, M. Anderson, C. Brinker, Chemistry of Materials 8 (1996) 1682.

[29] A. López, A. Ureña, J. Rams, Thin Solid Films 519 (2011) 7904.

[30] J. Hwang, B.I. Lee, V. Klep, I. Luzinov, Materials Research Bulletin 43 (2008) 2652.

[31] R. Lakshmi, T. Bharathidasan, B. Basu, Applied Surface Science 257 (2011) 10421.

[32] A. Kessman, D. Huckaby, C. Snyder, S. Kukureka, D. Cairns, Wear 267 (2009) 614. 
[33] E. Rabinowicz, L. Dunn, P. Russell, Wear 4 (1961) 345.

[34] A. Stalder, T. Melchior, M. Müller, D. Sage, T. Blu, M. Unser, Colloids and Surfaces A: Physicochemical and Engineering Aspects 364 (2010) 72.

[35] W. Oliver, G. Pharr, Journal of Materials Research 7 (1992) 1564.

[36] F. Ecarla, CSM Instruments Technical Workshop, PDF; Brescia, Italy (April 2012). 